\title{
High-Quality Perovskite Films for Efficient and Stable
} Light-Emitting Diodes
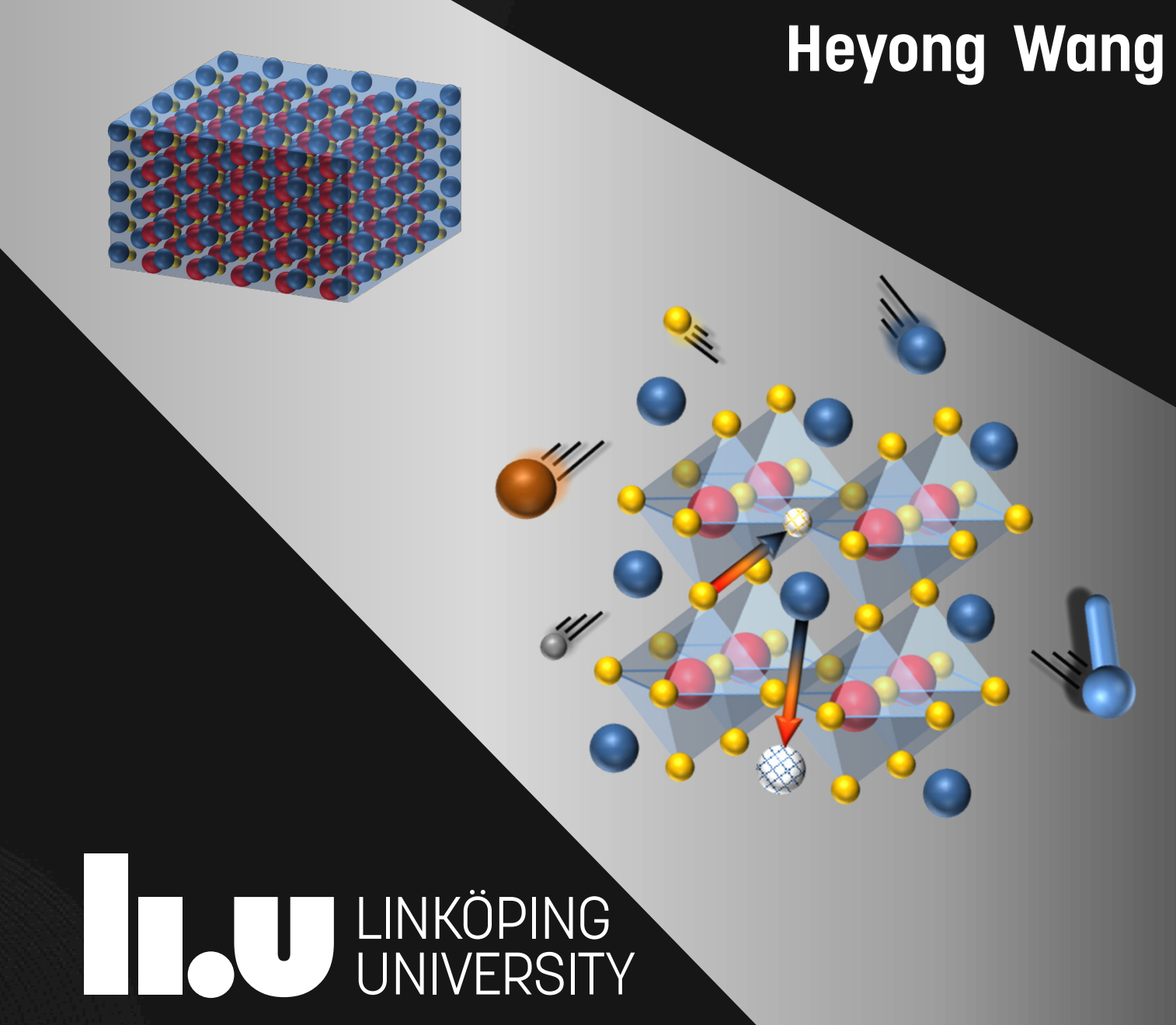


\title{
High-Quality Perovskite Films for Efficient and Stable Light-Emitting Diodes
}

\author{
Heyong Wang
}

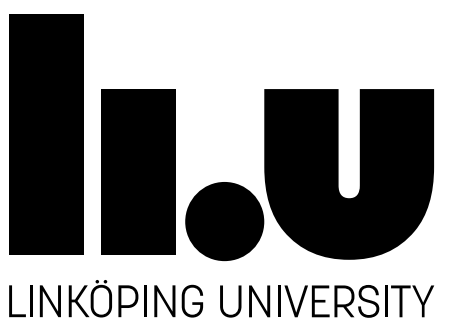

Electronic and Photonic Materials (EFM)

Department of Physics, Chemistry and Biology (IFM)

Linköpings universitet, SE-581 83 Linköping, Sweden

Linköping 2020 
Copyright (C) Heyong Wang, 2020

Printed in Sweden by LiU-Tryck, 2020

ISSN: 0345-7524

ISBN: 978-91-7929-813-5 


\section{Abstract}

Metal halide perovskites have attracted significant attention for lightemitting applications, because of their excellent properties, such as high photoluminescence quantum yields (PLQYs), good charge mobility, narrow emission bandwidth, readily tunable emission spectra ranging from ultraviolet to near-infrared, and solution processability. Since the first room-temperature perovskite-based light-emitting diodes (PeLEDs) reported in 2014, tremendous efforts have been made to promote the efficiencies of PeLEDs, including theoretical simulation, materials design, and device engineering. To reach the ultimate goal of commercialization, PeLEDs with both high-efficiency and long-term operational stability are desired. Achieving high-quality perovskite emissive films is key towards this goal. Centering around the high-quality perovskite films, in this thesis, we demonstrate effective synthesis strategies for the deposition of highquality perovskite films (including both three-dimensional and mixeddimensional perovskites) and investigate the effects of ion migration in the perovskite films on the performance of PeLEDs.

Due to the fast crystallization nature of perovskites and the low formation energy of defects, controlling the crystallization processes of these films has proved to be an effective approach for achieving highquality perovskite films. For three-dimensional (3D) perovskite films, we have controlled the formation of these films through the assistance of molecules with the amino group. Herein, we have chosen an electrontransport molecule with two amino groups, 4,4'-diaminodiphenyl sulfone (DDS), to control the crystallization process of perovskite films (Paper 1). The resulting perovskite films consists of in-situ formed high-quality perovskite nanocrystals embedded in the electron-transport molecular matrix, resulting in improved PLQYs and structural stability. PeLEDs based on these perovskite films have exhibited both high efficiency and long operational stability.

In addition, we have investigated the formation of mixed-dimensional perovskite films. Efficient PeLEDs based on mixed-dimensional perovskite films were fabricated with tin dioxide $\left(\mathrm{SnO}_{2}\right)$ as an electron transport layer (Paper 3). We also note that the deposition methods have a significant impact on the morphology and optical properties of prepared mixed-dimensional perovskite films (Paper 4). In addition, we provide an 
effective method to extend the deposition of mixed-dimensional perovskite films, replacing organic ammonium halides with amines in the perovskite precursor solutions to form organic spacer cations through the in-situ protonation process of amines (Paper 2).

In spite of these efforts, the performance of PeLEDs is still far from the commercialization standard, partially limited by ion migration. In Paper 5, we discuss impacts of mobile ions in the perovskite films on the performance of PeLEDs. We find that a dynamic redistribution of mobile ions can change current density of a device, leading to EQE/hysteresis during forward and reverse voltage scan and enhanced EQE under constant driving voltages. In addition, we have found that excess mobile ions in the perovskite layer can aggravate the hysteresis and shorten the operational stability of PeLEDs.

In this thesis, we also discuss the remaining key challenges in the PeLED field, including the achievement of high-performance blue, white, and lead-free PeLEDs, as well as possible strategies to address these challenges. We hope that our research findings provide insights into the basic science behind the perovskite materials, and broadly benefit other optoelectronic communities, such as perovskite solar cells, flexible electronics, and so on.

Keywords: perovskite light-emitting diodes, efficiency, stability, growth process, ion migration 


\section{Populärvetenskaplig Sammanfattning}

Metall-halid-perovskiter har fătt mycket uppmärksamhet för deras möjlighet att användas i ljusemitterande applikationer på grund utav utmärkta egenskaper så som högt fotoluminiscent kvantutbyte (PLQYs), god mobilitet, smalt emissionsband, lättjusterat emissionsspektra i intervallet ultraviolett till nära infrarött, samt för att de kan beredas med en lösningsbaserad process. Ända sedan de första perovskitbaserade ljusdioderna (PeLEDs) som verkande i rumstemperatur rapporterades 2014 har enormt arbete lagts ner på att öka effektiviteten hos PeLEDs, till exempel genom teoretiska simuleringar, materialdesign och teknisk utformning utav lysdioden. För att nå det slutgiltiga målet med kommersialisering behövs PeLEDs som har både hög effektivitet och driftstabilitet. Att uppnå högkvalitativa emitterande perovskitfilmer är nyckeln för att nå detta mål. I den här avhandlingen, vilken fokuserar på högkvalitativa perovskitfilmer, demonstrerar vi effektiva syntetiseringsstrategier för deponering utav högkvalitativa perovskitfilmer och undersöker effekterna jonmigration har på prestandan i perovskitfilmerna i PeLEDs.

På grund utav den snabbkristalliserande naturen hos perovskitfilmer och den låga bildningsenergin för defekter så har kontroll utav kristallisationsprocessen av dessa filmer visat sig vara en effektiv väg för att uppnå högkvalitativa perovskitfilmer. När det gäller tredimensionella perovskitfilmer så har vi kontrollerat bildandet av dessa filmer genom assistans från molekyler med aminogrupper. $\mathrm{Vi}$ har valt en elektrontransporterande molekyl som har två aminogrupper, 4,4'diaminodifenyl sulfon (DDS), för att kontrollera kristallisationsprocessen av perovskitfilmer (artikel 1). De beredda perovskitfilmerna bestod av insitu-bildade högkvalitativa perovskitnanokristaller inbäddade $\mathrm{i}$ en elektrontransporterande molekylär matris vilket resulterade i förbättrat fotoluminiscent-kvantutbyte och strukturell stabilitet. PeLEDs baserade på dessa perovskitfilmer har uppvisat både hög effektivitet och lång driftstabilitet.

Därtill har vi undersökt bildandet av blanddimensionella perovskiter. Effektiva PeLEDs baserade på blanddimensionella perovskiter var tillverkade med tenndioxid ( $\mathrm{SnO} 2$ ) som elektrontransportlager (artikel 3). Vi noterade även att val av deponeringsmetod hade signifikant effekt på 
morfologi och optiska egenskaper av de beredda blanddimensionella perovskitfilmerna (artikel 4). Därutöver tillhandahöll vi en effektiv metod för att deponera blanddimensionella perovskitfilmer genom att ersätta de vanligen använda organiska ammoniumhaliderna med aminer i prekursorlösningen för att bilda organiska distansierande katjonfilmer genom en in-situ-protoneringsprocess av aminer (artikel 2).

Även om PeLEDs med högkvalitativa perovskitfilmer har visat förbättrad effektivitet och driftstabilitet så uppvisar lysdioderna fortfarande egenskaper som associeras med jonmigration. I artikel 5 diskuterar vi effekten av mobila joner i perovskitfilmer och hur de påverkar PeLEDs prestanda. Vi fann att den dynamiska omfördelningen av mobila joner i perovskitskiktet kan ändra det elektriska nettofältet $\mathrm{i}$ perovskitskiktet, modifiera laddningsbärarnas rekombination i perovskitskiktet och leda till ostadig strömdensitet. Dessutom har vi funnit att överskott av mobila joner i perovskitskiktet är en av de största anledningarna till den korta driftstabiliteten för PeLEDs.

I denna avhandling diskuterar vi även kvarstående nyckelproblem i PeLED-fältet så som bedriften att uppnå högpresterande blå, vita och blyfria PeLEDs så väl som möjliga strategier att möta dessa utmaningar. Våra forskningsresultat ger insikter om den grundläggande vetenskapen bakom perovskitmaterialen vilka vi tror kommer vara till stort gagn för andra optoelektroniska fält, så som perovskitsolceller, flexibel elektronik och så vidare.

Nyckelord: perovskit-lysdioder, effektivitet, stabilitet, tillväxtprocess, jonmigration 


\section{Acknowledgement}

Nothing of this could have been accomplished without the kind support and tremendous help from my supervisors, colleagues, friends, and families. Everyone has contributed their help to the person that I am today, and for that, I am very thankful.

Firstly, I owe my great thankfulness to my supervisor Prof. Feng Gao for accepting me as a PhD student here at Linköping University. Thank you to give me the chance of staying in academia. Also, thank you to give me enough freedom, tremendous help, and great suggestions on my work and career.

My great thankfulness also gives to my co-supervisors Prof. Olle Inganas and Dr. Xiao-Ke Liu. Thank you, Olle, for the discussions with me. Your creative questions and comments always inspire me to give a second thought on my work from a new point of view. Thank you, Xiao$\mathrm{Ke}$, for your endless help from operating the glovebox to writing the manuscript. You are a good supervisor, a good teacher, and a good friend.

I would like to give my special gratitude to Dr. Zhongcheng Yuan and Ms. Hongling Yu. We work together from the beginning of our lab and gain plenty of joyful time. Thank you, Zhongcheng, for your help in the lab and good suggestions on the lab work. Thank you, Hongling, for your help both in the work and daily life. I will never forget the first day when I arrived at Linkoping. With your help, we bought the first and urgent things we need.

I also want to acknowledge all my collaborators from over the world: Prof. Caterina Ducati's group and Prof. Richard H. Friend's group from University of Cambridge, UK. Prof. Yizheng Jin's group from Zhejiang University, China. Prof. Sabina Abbrent's group from Institute of Macromolecular Chemistry of the Czech Academy of Sciences, Czech Republic. Prof. Yue Lu's group from Beijing University of Technology, China. Prof. Mats Fahlman's group, Prof. Galia Pozina, Dr. Chunxiong Bao, Dr. Zhangjun Hu, and Dr. Jian Qingfrom Linköping University, Sweden. Thanks for all your help on the measurements, great ideas, and discussions.

I also would like to express my thanks to my colleagues, David Hardy, Julia Morat and Max Karlsson. Thank you very much for your kind help with my thesis writing. 
My sincere thanks also give to all my friends in Sweden and China. Thank you for the joyful time that we have spent over the last four years.

I am also very grateful to the following department staff: AnnaMaria Uhlin, Ann-Charlotte Svensson Holm, Chunxia Du, Thomas Lingefelt, Rickard Liljedahl, and Sven Andersson, who make my research much easier than I thought.

At last, I must thank my parents and my wife Ge Liu for your love and support. My beloved wife, I cannot tell you how thankful I am for you. Thank you for being with me.

Heyong Wang

August 2020 at Linköping University 


\section{List of publications included in the thesis}

1. Perovskite-molecule composite thin films for efficient and stable light-emitting diodes

Heyong Wang, Felix Utama Kosasih, Hongling Yu, Guanhaojie Zheng, Jiangbin Zhang, Galia Pozina, Yang Liu, Chunxiong Bao, Zhangjun Hu, Xianjie Liu, Libor Kobera, Sabina Abbrent, Jiri Brus, Yizheng Jin, Mats Fahlman, Richard H Friend, Caterina Ducati, Xiao-Ke Liu, Feng Gao: Nature communications, 2020, 11: 891

2. High-quality Ruddlesden-Popper perovskite films based on in situ formed organic spacer cations

Jian Qing, Chaoyang Kuang, Hevong Wang, Yuming Wang, Xiao-Ke Liu, Sai Bai, Mingjie Li, Tze Chien Sum, Zhangjun Hu, Wenjing Zhang, Feng Gao: Advanced Materials, 2019, 31(41): 1904243.

3. Efficient perovskite light-emitting diodes based on a solutionprocessed tin dioxide electron transport layer

Hevong Wang, Hongling Yu, Weidong Xu, Zhongcheng Yuan, Zhibo Yan, Chuanfei Wang, Xianjie Liu, Mats Fahlman, Junming Liu, Xiao-Ke Liu, Feng Gao: Journal of Materials Chemistry C, 2018, 6(26): 6996

4. Efficient light-emitting diodes based on in-situ self-assembled perovskite nanocrystals

Heyong Wang, Hongling Yu, Xiao-Ke Liu, Feng Gao: Journal of Photonics for Energy, 2018, 8(4): 046002

5. Dynamic ion redistribution in perovskite light-emitting diodes

Heyong Wang, Zhan Chen, Jingcong Hu, Hongling Yu, Chaoyang Kuang, Jiajun Qin, Xianjie Liu, Yue Lu, Mats Fahlman, Lintao Hou, Xiao-Ke Liu and Feng Gao: Submitted 2020

\section{Author contribution}

Paper 1, 3-5: I fabricated the perovskite devices, carried out most of the characterizations and data analysis, and wrote the first draft of the manuscript.

Paper 2: I performed the scanning electron microscope and tappingmode atomic force microscopy measurements and associated data analysis. 


\section{List of publications not included in the thesis}

\section{Ultra-High Vacuum Annealing-Assisted Quantum Wells Dimensional Tailoring for Perovskite Light-Emitting Diodes Efficiency Enhancement}

Yong Yu, Heyong Wang, Weidong Xu, Chaoyang Kuang, Fuxiang Ji, Slawomir Braun, Xianjie Liu, Chang Yi, Feng Gao, Mats Fahlman: ACS Applied Materials \& Interfaces, 2020, 12, 24965

2. Efficient and Tunable Electroluminescence from In Situ Synthesized Perovskite Quantum Dots

Hongling Yu, Hevong Wang, Jiangbin Zhang, Jun Lu, Zhongcheng Yuan, Weidong Xu, Lars Hultman, Artem A Bakulin, Richard H Friend, Jianpu Wang, Xiao-Ke Liu, Feng Gao: Small, 2019, 15(8): 1804947.

3. Rational molecular passivation for high-performance perovskite light-emitting diodes

Weidong Xu, Qi Hu, Sai Bai, Chunxiong Bao, Yanfeng Miao, Zhongcheng Yuan, Tetiana Borzda, Alex J Barker, Elizaveta Tyukalova, Zhangjun Hu, Maciej Kawecki, Hevong Wang. Zhibo Yan, Xianjie Liu, Xiaobo Shi, Kajsa Uvdal, Mats Fahlman, Wenjing Zhang, Martial Du-champ, Jun-Ming Liu, Annamaria Petrozza, Jianpu Wang, Li-Min Liu, Wei Huang, Feng Gao. Nature Photonics, 2019, 13(6): 418 


\section{Contents}

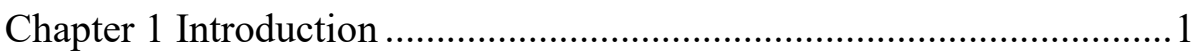

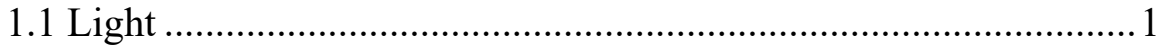

1.2 Light-Emitting Diodes........................................................... 2

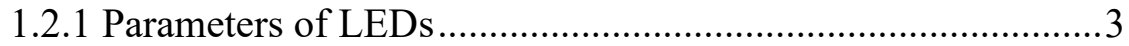

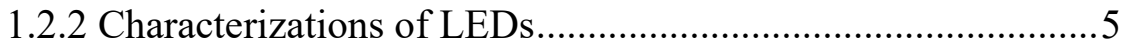

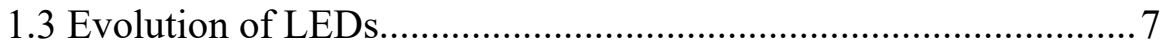

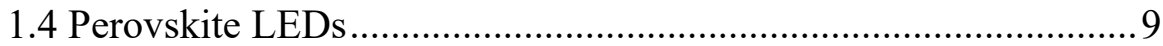

Chapter 2 High-Efficiency Perovskite Light-Emitting Diodes...............13

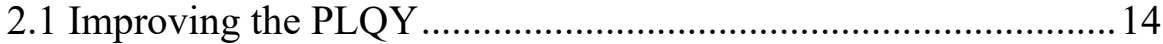

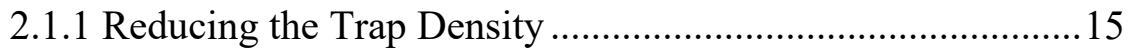

2.1.2 Enhancing the Radiative Recombination ............................ 17

2.1.3 Suppressing the Three-Body Auger Recombination ..............18

2.2 Optimizing the Injection Efficiency .......................................... 18

2.2.1 Underlying Charge-Transport Layers and Interfacial Layers 18

2.2.2 Interfacial Layers on Top of Perovskite ................................ 19

2.3 Improving the Light Outcoupling Efficiency .............................. 19

Chapter 3 High-Quality Perovskite Films...........................................21

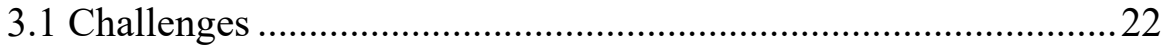

3.1.1 Large Perovskite Grain Sizes ...........................................22

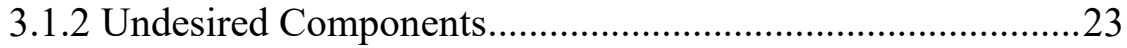

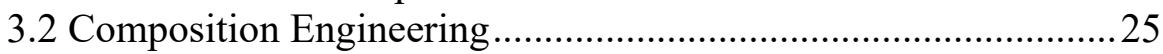

3.2.1 Non-Stoichiometric Perovskite Precursors ............................25

3.2.2 Large-Sized Organic Ammonium Salts................................26

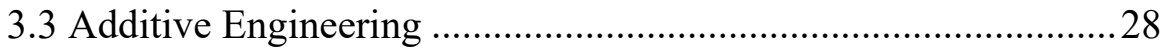

3.3.1 Additive-Assisted Crystallization ........................................28

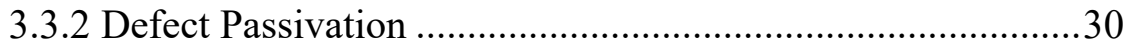

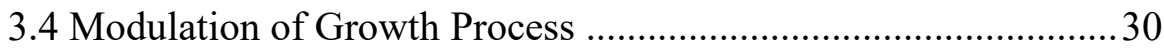

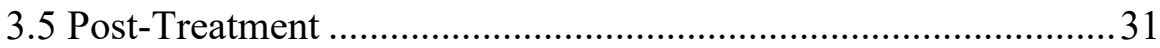

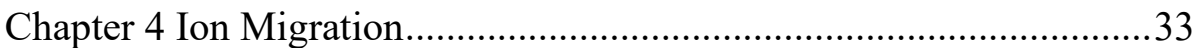

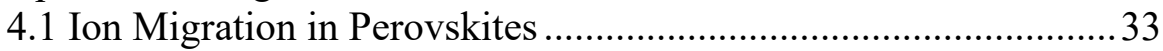

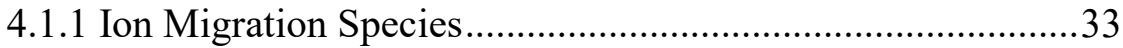

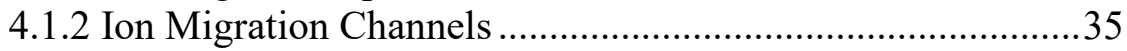

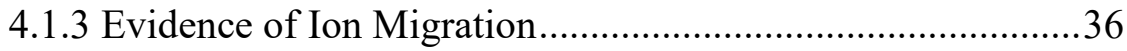

4.2 Ion Migration Induced Phenomena in PeLEDs............................ 38

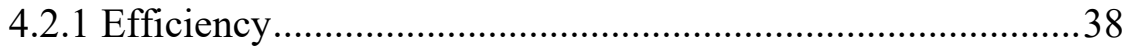

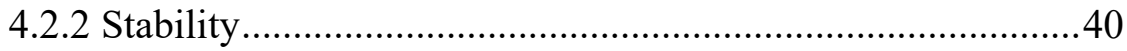


Contents

4.3 Suppress Ion Migration.......................................................... 41

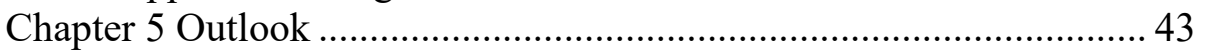

5.1 High-Performance Blue PeLEDs............................................. 43

5.2 White PeLEDs ....................................................................... 44

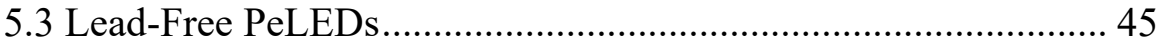

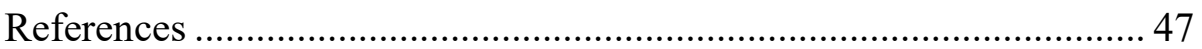




\section{Chapter 1 Introduction}

\subsection{Light}

In physics, the term light refers to electromagnetic radiation of any wavelength, as shown in Fig. 1.1, ${ }^{1}$ which can be divided into $\gamma$-ray, Xray, ultraviolet light, visible light, infrared light, microwave and radio waves. In our daily lives, when we talk about light, it mainly means visible light (400-700 $\mathrm{nm}$ ), which can be perceived by human eyes.

One of the basic and important features of light is wave-particle duality property. It propagates as waves, as shown in the famous doubleslit experiment. ${ }^{2}$ Light is generally described as a wave, using wavelength or frequency, which indicates the extensibility in space and time. In the meanwhile, the energy imparted by the waves can be absorbed like the way particles are absorbed, indicating the particle property. The absorbed energy of the light is called a photon and represents a quanta of light. The photon energy equation (Equation 1.1) describes the relationship between wave property and particle property.

$$
E=h f=h c / \lambda
$$

where $E$ is the energy of the photon; $h$ is Planck constant; $c$ is the speed of light in the medium; $f$ and $\lambda$ is the frequency and wavelength of light, respectively.

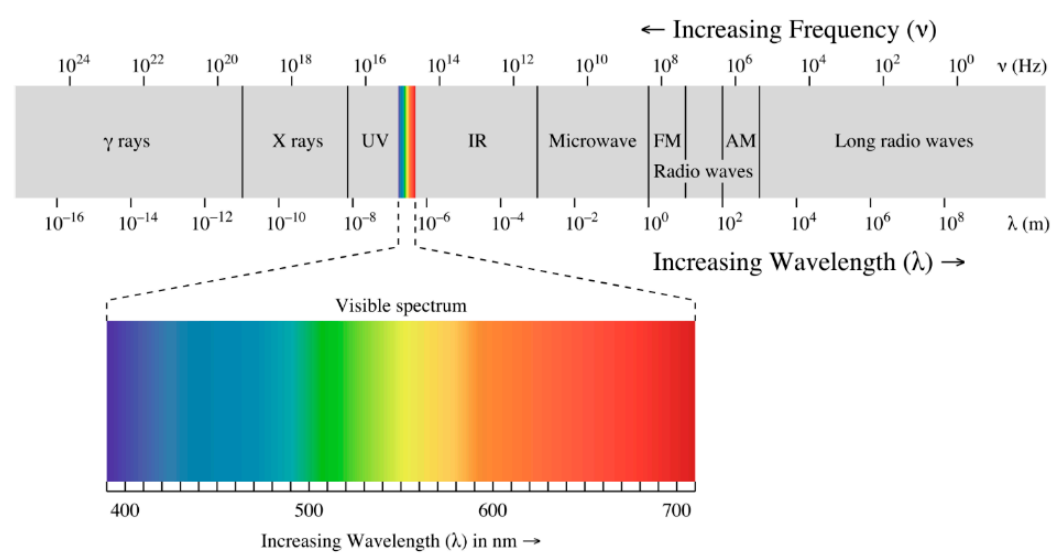

Figure 1.1 The electromagnetic spectrum with the visible portion highlighted. ${ }^{1}$ 
The main source of light on earth is sunlight, which mainly includes infrared, visible, and ultraviolet light. It provides energy for plants and other autotrophic organisms to convert into chemical energy, which can be used to synthesize carbohydrates and to fuel the organisms' activities. Bioluminescence generated by some species of animals is another kind of light source. For example, fireflies use light to locate mates, and vampire squids use it to hide themselves from prey. Historically, another important light source for humans has been fire, from ancient campfires to modern kerosene lamps. With the development of electric light sources and power systems, electric lighting has replaced firelight, being the main light source for humans.

\subsection{Light-Emitting Diodes}

Light-emitting diodes (LEDs), as a new-generation solid-state electric light sources, are increasingly popular in modern society. Compared with other light sources, including incandescent and fluorescent lamps, LEDs have the advantages of high energy efficiency, lightweight, robustness and long lifetime. ${ }^{3}$

Generally, LEDs are tiny devices made from semiconductor materials. As shown in Fig. 1.2, under proper forward-biased conditions, current is injected and flows through the LED. Photons of various wavelengths are subsequently emitted from the emission region. This process is commonly known as electroluminescence (EL). To quantitatively estimate the performance of an LED, the photons is detected by an optical
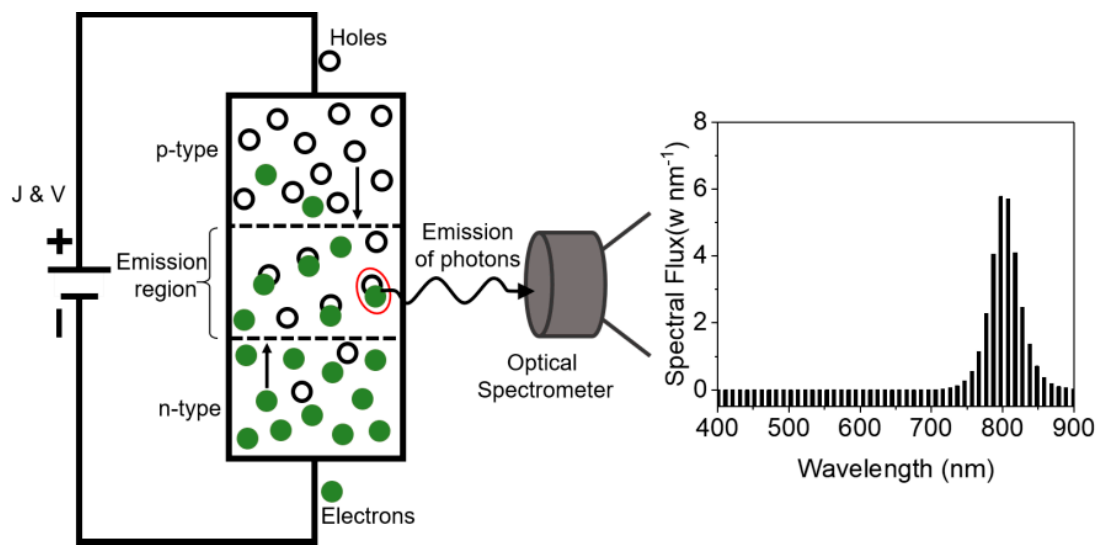

Figure 1.2 The working principle and measurement of an LED. 
spectrometer, which is used to separate and measure individual photons. Derived from the current, voltage, and detected photons, various parameters of LEDs can be calculated.

\subsubsection{Parameters of LEDs}

In this section, measuring by an integrating sphere coupled with a spectrometer, several parameters of a planar LEDs with ideal Lambertian emission are discussed.

Radiance $\left(\boldsymbol{L}_{e, \Omega}\right)$. Spectral radiance is the radiant flux emitted by a given surface of an LED per unit solid angle per unit projected area with the SI unit of watt per steradian per square metre $\left(\mathrm{W} \mathrm{sr}^{-1} \mathrm{~m}^{-2}\right)$. It directly corresponds to the power of emitted photons. The $L_{e, \Omega}$ can be defined as Equation 1.2

$$
L_{e, \Omega}=\frac{\partial^{2} \Phi_{e}}{\partial \Omega \partial A \cos \theta}=\frac{1}{\pi A} \int \Phi_{e, \lambda} d \lambda
$$

where $\Phi_{e}$ is the total emitted radiant flux; $\Phi_{e, \lambda}$ is the emitted radiant flux at a certain wavelength $\lambda ; \Omega$ is the solid angle; $A$ is the active emitting area of LEDs; $A \cos \theta$ is the projected area.

Luminance $\left(\boldsymbol{L}_{V}\right)$. Luminance (with the SI unit of $\mathrm{cd} \mathrm{m}^{-2}$ ) is the luminous flux of visible light emitted by a given surface of an LED per unit solid angle per unit projected area. Luminance is a special parameter related to the human visual perception of brightness, calculated by Equation 1.3

$$
L_{V}=\frac{k}{\pi A} \int \Phi_{e, \lambda} \cdot V_{(\lambda)} d \lambda
$$

where $k$ is a constant value of $683 \mathrm{~lm} \mathrm{~W}^{-1} ; V_{(\lambda)}$ is photopic luminosity function.

Internal Quantum Efficiency (IQE). IQE $\left(\eta_{\text {int }}\right)$ is a ratio of the number of total photons generated by an LED to the number of total injected carriers. The value of IQE is related to radiative efficiency $\left(\eta_{\mathrm{rad}}\right)$ and injection efficiency $(\gamma)$, defined as Equation 1.4

$$
\eta_{\text {int }}=\eta_{\text {rad }} \times \gamma
$$

$\eta_{\text {rad }}$ describes the probability of radiative recombination of electronhole pairs, approximatively equal to photoluminescence quantum yield (PLQY), which is one of the intrinsic properties of an emissive material. 
The injection efficiency is determined by the LED structure, which is dependent on injection balance of electrons and holes. $\gamma=1$ means that all the injected electrons and holes can recombine. Equation 1.4 indicates that both emissive materials and device structures are important to realize high-efficiency LEDs.

External Quantum Efficiency (EQE). Obviously, for LED applications, what matters is the amount of light extracted outside the devices. EQE $\left(\eta_{\text {ext }}\right)$ is such a parameter. It is a ratio of the number of photons extracted out of an LED in the viewing direction to the number of total injected electrons in the device. It is related to the IQE and light outcoupling efficiency $\left(\eta_{o c}\right)$, defined as Equation 1.5

$$
\eta_{\text {ext }}=\eta_{\text {int }} \times \eta_{o c}
$$

where $\eta_{o c}$ is an optical term, independent of electrical phenomena.

Based on the measurement through an integrating sphere and spectrometer system, EQE can be calculated by Equation 1.6

$$
\eta_{\text {ext }}=\frac{e}{h \cdot c \cdot I} \int \Phi_{e, \lambda} \cdot \lambda d \lambda \times 100 \%
$$

where $e$ is the elementary charge; $I$ is the device current flowing through the LEDs.

Wall-Plug Efficiency. Wall-plug efficiency $\left(\eta_{\text {wp }}\right)$ is a energy conversion efficiency of an LED with the unit of percentage (\%). It is the ratio of the total light output power (total emitted radiant flux, $\Phi_{e}$, with unit of W) to the electrical input power $(I \bullet V$, with unit of $\mathrm{W})$, derived from Equation 1.7

$$
\eta_{w p}=\frac{\int \Phi_{e, \lambda} d \lambda}{I \cdot V} \times 100 \%
$$

Luminous Power Efficiency. For LED applications in display and lighting, luminous power efficiency $\left(\eta_{p}\right)$ is to show how much visible light produced by a certain input electrical power with the unit of lumens per watt $\left(\mathrm{lm} \mathrm{W}^{-1}\right)$. Compared with the wall-plug efficiency, here, the light output power is replaced with the luminous flux (with the unit of $1 \mathrm{~m}, 1 \mathrm{~lm}$ $=1 \mathrm{~cd} \mathrm{sr}$ ). Therefore, the $\eta_{p}$ can be derived from Equation 1.8 


$$
\eta_{p}=\frac{k \cdot \int \Phi_{e, \lambda} \cdot V_{(\lambda)} d \lambda}{I \cdot V}
$$

Luminous efficiency. Luminous efficiency $\left(\eta_{L}\right)$ is also called current efficiency with the unit of candelas per ampere $\left(\mathrm{cd} \mathrm{A}^{-1}\right)$. It is another commonly used parameter to assess the performance of visible LEDs, derived from Equation 1.9

$$
\eta_{L}=\frac{k \cdot \int \Phi_{e, \lambda} \cdot V_{(\lambda)} d \lambda}{\pi \cdot I}
$$

\subsubsection{Characterizations of LEDs}

To measure the performance of an LED, we need to simultaneously measure the electric parameters (applied voltage and current) and the emitted light. During the measurement, an LED is generally driven by a Keithley electrometer, which can provide and record the applied voltages and currents as shown in Fig. 1.3a. For the collection of the emitted light, there are two widely used systems, a silicon photodiode coupled with a

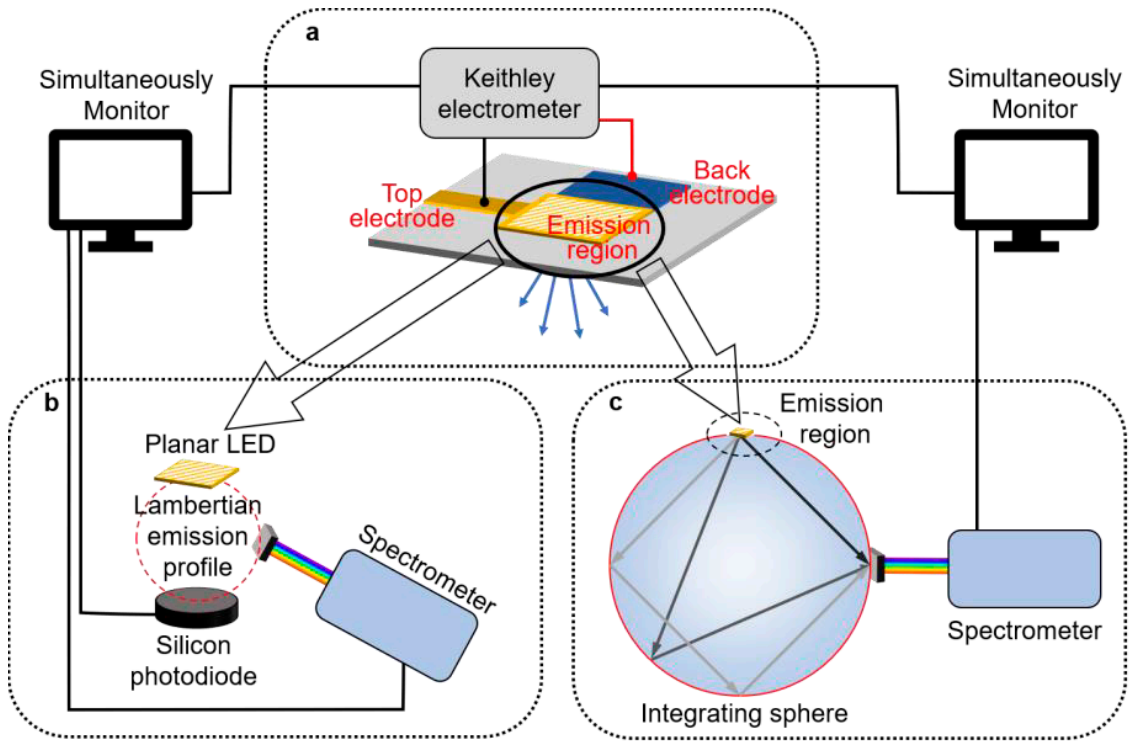

Figure. 1.3 Schematic of LED characterization systems. (a) LEDs driven by a Keithley electrometer. Photons are collected by (b) a silicon photodiode and a spectrometer, which collect EL intensity and spectra, respectively, or (c) an integrating sphere coupled with a spectrometer, which simultaneously collects EL intensity and spectra. 
spectrometer (Fig. 1.3b) or an integrating sphere coupled with a spectrometer (Fig. 1.3c). ${ }^{4-8}$

For a planar LED that has the Lambertian emission profile, the emitted light can be collected by a silicon photodiode as shown in Fig. 1.3b. The silicon photodiode is placed perpendicularly to the device's emitting surface and collects the photons from the working LED. The spectrometer is used to record the EL spectra. Combing with the parameters of distance (between the silicon photodiode and the device surface) and solid angle of the silicon photodiode about the surface normal (for the collection of light), the performance of the working LED can be calculated through the recorded electric and optical parameters.

An integrating sphere coupled with a spectrometer is another light collection system as shown in Fig. 3c. The LED is in close contact with the input port of the integrating sphere and has a smaller emission area than that of the input port of the integrating sphere. Hence, we can assume that all the lights emitted from the forward view are collected. The collected light goes into the spectrometer, which separates the light into individual photons and then counts these photons. The performance of the LED can be calculated from the recorded electric parameters and photons (energy and counts).

The EQEs, radiances, and EL spectra of LEDs studied in this thesis are measured by a homemade system (an integrating sphere coupled with a spectrometer). The operational stability of LEDs is measured through a silicon photodiode. Both systems are stored in a nitrogen filled glovebox, which can protect LEDs from oxygen and humidity. 


\subsection{Evolution of LEDs}

EL is a result of radiative recombination of electrons \& holes (or excitons) in a semiconductor material. The mechanisms behind the emission of photons in LEDs lie in the energy band theory of solids. The process of EL can be described by an analogous mechanism to that of PL, as shown in Fig. 1.4, where the materials are excited electrically and the electrons relax to ground state (de-excitation) radiatively. ${ }^{9}$ In this section, we present a brief introduction from a materials point of view.

Electroluminescence was first discovered by Henry Joseph Round as early as 1907; he noticed emission of yellowish light by applying a potential of $10 \mathrm{~V}$ to an inorganic semiconductor (carborundum, silicon carbide) crystal. ${ }^{10}$ More detailed experiments were presented by Lossev, whose work spanned from the 1920 s to the 1930s. ${ }^{11}$ In the $1950 \mathrm{~s}$, both academic and industrial communities carried out many experiments to generate light emission at $p-n$ junctions of diodes. ${ }^{12}$ Since the semiconductors used at that point had indirect band gaps, their efficiencies were very limited. Much higher quantum efficiencies were subsequently reported from direct-bandgap GaAs in $1962 .{ }^{13}$ After that, it was considered imperative to use direct bandgap material for efficient electroluminescence. The subsequent studies on the direct-bandgap materials had a profound impact on commercial LEDs. In 1972, M. George Craford at Monsanto Company used one red and one green gallium phosphide chips to make yellow LEDs. Incidentally, the Monsanto Company is the first company, which produced LED lights on a large scale and for mass consumption. Continuous and intensive efforts into LED technology led to a development of the first generation of superbright red, yellow, and green LEDs with indium gallium aluminum phosphide as semiconductor materials in the early years of the 1980s. In

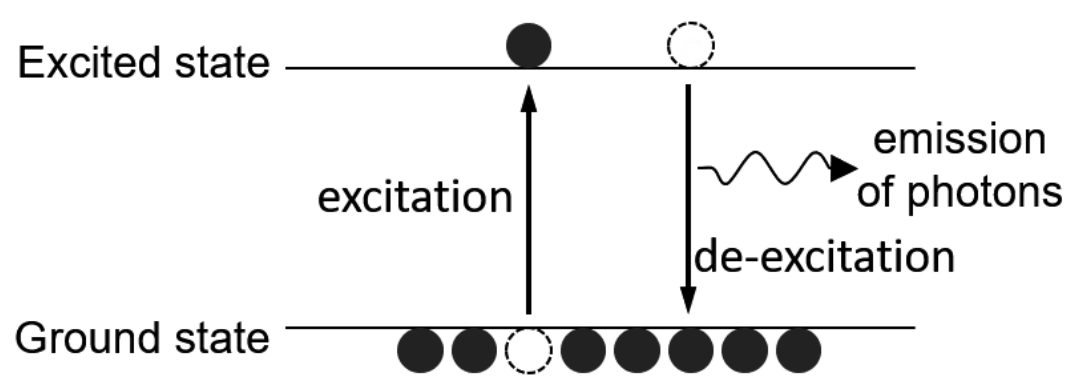

Figure 1.4 Mechanism of luminescence of semiconductor materials. 
1994, Shuji Nakamura invented the ultra-bright blue LEDs using gallium nitride; afterwards, indium gallium nitride was used to develop highintensity blue and green LEDs. These ultra-bright blue LEDs led the foundation for the development of cost-effective and high-performance white LED lights that are now commonly used for lighting. Owing to "the invention of efficient blue light-emitting diodes which has enabled bright and energy-saving white light sources", the Nobel Prize in Physics in 2014 was awarded jointly to Isamu Akasaki, Hiroshi Amano and Shuji Nakamura.

Organic light-emitting diodes (OLEDs) based on organic emissive materials have been considered as one of the most promising technologies for future displays and lighting since the breakthrough made by Tang and VanSlyke from Kodak in $1987 .{ }^{14}$ A number of organic materials have been developed in order to fulfill the requirements of high-performance OLEDs, including small molecules and polymers. Small molecule- (SMOLEDs) and polymer-based LEDs (PLEDs) are usually fabricated through vacuum deposition and solution process, respectively. In addition, according to the luminescence mechanism, the evolution of OLEDs can be divided into fluorescent materials, phosphorescent materials, delayed fluorescence, and doublet emission. ${ }^{15,16}$ Recently, OLEDs are already commercially applied in smartphone and TV displays. ${ }^{17}$

Colloidal quantum dots (CQDs) have emerged as a new class of semiconducting materials for display applications due to their excellent optical properties, including tunable bandgap, narrow emission bandwidth, and high efficiency. ${ }^{18}$ CQDs are nanometer-sized inorganic semiconductor particles, where excitons can form since the particle size is smaller than their Bohr diameter. CQDs' small size also enables them to be synthesized and suspended in the solution, facilitating a range of solution-based processing methods, including spin-coating, inkjet printing, and roll-to-roll casting. ${ }^{19}$ The first route to successful synthesis of CQDs was based on aqueous and ionic chemistry, developed by Brus and Henglein in the $1980 \mathrm{~s}^{20-22}$ To produce monodisperse and highquality CQDs, a synthetic scheme of the pyrolysis of organometallic precursors with coordinating ligands in an organic solvent (referred to as the hot-injection method) was reported in $1993 .{ }^{23}$ This approach was a milestone in the development of the synthesis of high-quality CQDs and enabled their widespread investigations and applications. This approach also led to the concept of size-focusing, proposed in 1998 by Alivisatos and coworkers, ${ }^{24}$ contributing to the deep understanding of nanocrystal 
growth and rational synthesis of CQDs. A further major step toward the preparation of stable and highly luminescent CQDs was made by Hines and Guyot-Sionnest, who passivated the surface using an inorganic wider-bandgap semiconductor capping shell (core/shell CQDs). ${ }^{25}$ $\mathrm{CdSe} / \mathrm{ZnS}, \mathrm{CdSe} / \mathrm{CdS}, \mathrm{CdSe} / \mathrm{ZnSe}$ and $\mathrm{InP} / \mathrm{ZnS}$ core/shell CQDs have been subsequently synthesized, exhibiting improved PLQY of 50-90\%. ${ }^{25-}$ ${ }^{29}$ Besides the most studied core/shell type of heterostructure, a variety of other complex heterostructures have been developed, including dumbbells and tetrapods. ${ }^{30-32}$ In addition to the II-VI semiconductor CQD systems, IV-VI and III-V semiconductor CQDs were also synthesized with narrow size distributions and high PLQYs. ${ }^{33-35}$

\subsection{Perovskite LEDs}

In addition to the above-mentioned materials, metal halide perovskites have presented new opportunities toward low-cost and high-performance LED technology. These materials have demonstrated tunable emission spectra covering the whole visible range and narrow line shapes with the full width at half maximum (FWHM) in the range of 5-40 nm, resulting in high color purity and wide color gamut. Displays made from perovskite-based LEDs (PeLEDs) can show color space 140\% broader than the National Television System Committee standard and close to 100\% of the new International Telecommunication Union Rec. 2020 standard, making them promising in the application of ultra-high-definition displays. ${ }^{36}$ Meanwhile, due to the low formation energy and low Young's modulus, metal halide perovskite films exhibit "soft" nature, making them possible in the application of low-temperature-processed flexible devices. 
Metal halide perovskites are a rich class of materials sharing the classical perovskite crystal structure with a general formula of $\mathrm{ABX}_{3}$ (Fig. 1.5), where $\mathrm{A}$ is a monovalent cation, $\mathrm{B}$ is a divalent metal, and $\mathrm{X}$ is a halide anion. Metal halide perovskites were first reported in $1893,{ }^{37}$ upon which light-emitting devices and transistors were demonstrated during the 1990s. However, metal halide perovskites have not drawn much attention until they were employed in solar cells as light harvesters in $2009,{ }^{38}$ and in room-temperature LEDs as emissive layers in $2014^{7}$. Since then, various investigations on materials and devices have been carried out.

$\mathrm{CH}_{3} \mathrm{NH}_{3} \mathrm{PbI}_{3}\left(\mathrm{MAPbI}_{3}\right)$ has been the benchmark optoelectronic perovskite since its report as light harvesters in $2009 .{ }^{38}$ Based on this material, intensive investigations were carried out on crystals and films preparation, property investigations, and device fabrication. However, the volatile nature of the MA cation causes instability issue of $\mathrm{MAPbI}_{3}$ in the conditions of moisture, heat, and light soaking. To resolve this issue, derivatives of $\mathrm{MAPbI}_{3}$ were developed. Replacing MA with other organic or inorganic cations were attempted, resulting in improved stability and tunable bandgap. ${ }^{39,40}$ However, limited by Goldschmidt tolerance factor (Equation 1.10) and octahedral factor (Equation 1.11), A-site cations are mostly cesium $\left(\mathrm{Cs}^{+}\right)$, formamidinium $\left(\mathrm{CH}\left(\mathrm{NH}_{2}\right)_{2}{ }^{+}, \mathrm{FA}^{+}\right)$, or mixtures of such cations.

$$
t=\frac{r_{A}+r_{X}}{\sqrt{2}\left(r_{B}+r_{X}\right)}
$$

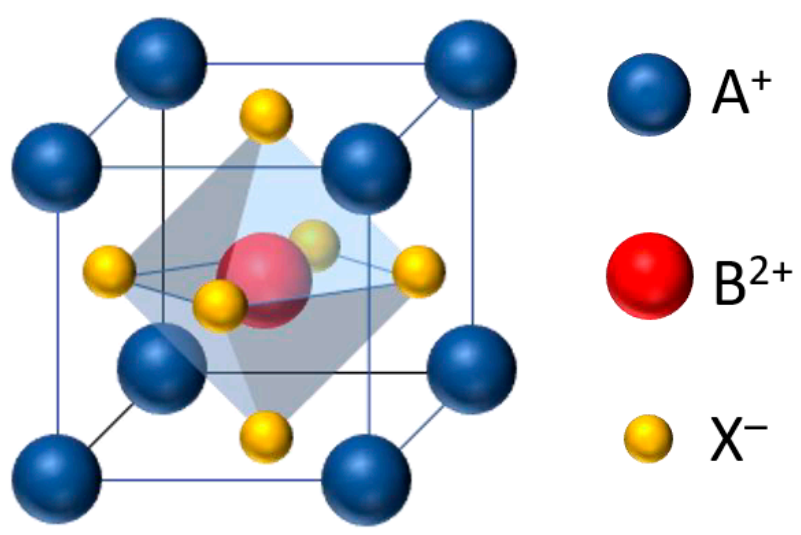

Figure 1.5 Diagram of the perovskite crystal structure 


$$
\mu=r_{B} / r_{X}
$$

In 2014, the first room temperature PeLEDs were reported with $\mathrm{MAPb}(\mathrm{Br} / \mathrm{I} / \mathrm{Cl})_{3}$ as emissive layers. ${ }^{7}$ Despite the poor performance associated with ionic defects and small exciton binding energy in such bulk perovskites, this pioneering work sparked the interest of using perovskites for LEDs. After that, a wide range of investigations on component-, size-, and dimensional-engineering resulted in different metal halide perovskites for LED applications. Benefiting from the established experience in both perovskite materials and solutionprocessed optoelectronic devices, the community has been able to quickly boost the EQEs of PeLEDs to over 20\% within several years, making them one of the hottest research topics.

In the following chapters of this thesis, we first discuss the basic requirements for achieving high-efficiency PeLEDs. In Chapter 2, we review the progress for achieving high-efficiency PeLEDs from basic parameters, including PLQY of perovskite emitters, injection efficiency, and light outcoupling efficiency.

To achieve efficient and stable PeLEDs, high-quality perovskite films are critically important. In Chapter 3, we outline the key challenges lying in preparation of high-quality perovskite films for high-performance PeLEDs. Meanwhile, we discuss several effective synthetic strategies to tackle these challenges, including composition engineering, additive engineering, modulation of growth process, and post-treatment of crystallized perovskite films.

In spite of high-quality perovskite films, PeLEDs still exhibit some special phenomena, which may be associated with ion migration. In Chapter 4, we introduce the origin and evidence of ion migration in perovskite materials and discuss the influence of ion migration on the performance of PeLEDs. Possible approaches that can suppress the ion migration process are also discussed.

In Chapter 5, we discuss the critical challenges remaining in the PeLEDs field, such as high-performance blue, white, and lead-free PeLEDs. In addition, we discuss possible strategies to address these challenges. 



\section{Chapter 2 High-Efficiency Perovskite Light- Emitting Diodes}

Perovskite light-emitting diodes (PeLEDs) have received increasing attention since $2014 .^{7}$ Benefiting from tremendous efforts in this field and rich experience from perovskite solar cells, the external quantum efficiencies (EQEs) of PeLEDs have increased very quickly in the past several years, reaching high values of over $20 \%{ }^{4-6,8,41,42}$ The rapid development of PeLEDs has demonstrated great promise towards a new generation of low-cost and high-performance LED technologies.

Generally, PeLEDs have a planar structure, where the perovskite emissive layer (EML) is sandwiched between a $n$-type electron transport layer (ETL) and a $p$-type hole transport layer (HTL). The anode and cathode contact the ETL and HTL, respectively. As shown in Fig. 2.1, PeLEDs can be fabricated by using $n-i-p$ and $p-i-n$ structures, depending on the deposition sequence of each layer. For both device structures, to achieve high-efficiency PeLEDs, several basic parameters should be considered, including photoluminescence quantum yield (PLQY) of perovskite emitters, injection efficiency, and light outcoupling efficiency. In this chapter, we discuss the development of high-efficiency PeLEDs from both materials and devices points of view.
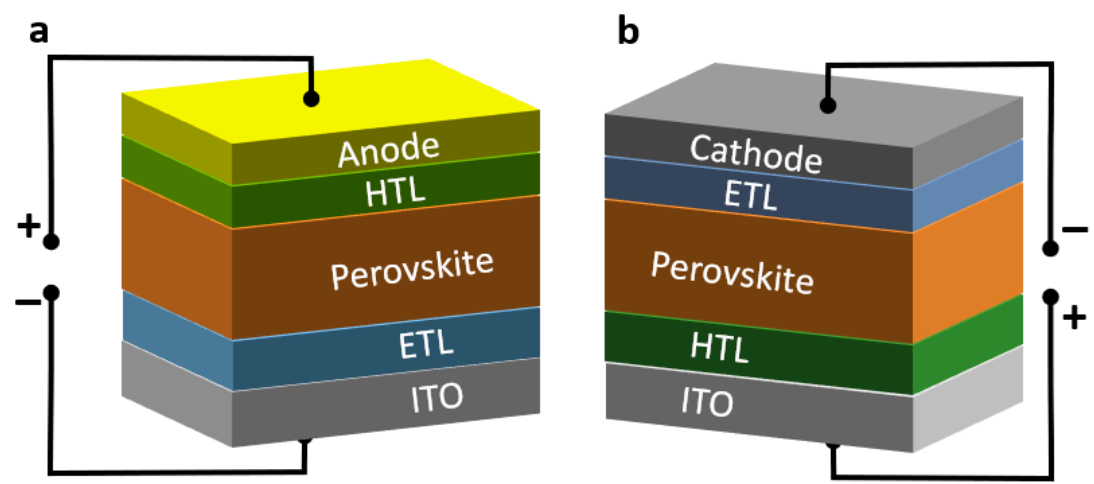

Figure 2.1 Typical device architectures of PeLEDs. (a) $n-i-p$ and (b) $p-i-n$ structures. 


\subsection{Improving the PLQY}

Achieving emissive materials with high PLQYs is a basic requirement for any high-efficiency LEDs. To achieve high PLQYs, it is necessary to maximize the radiative recombination rates and minimize the nonradiative recombination pathways. In semiconductor materials, nonradiative recombination generally includes trap-assisted recombination and three-body Auger recombination, which are linear and cubic to the concentration of carriers, respectively. Depending on the size and dimensionality of the perovskites, the radiative recombination can be bimolecular or excitonic, which are square and linear to the concentration of carriers, respectively. Therefore, the PLQYs can be quantified by Equation 2.1 and 2.2 for perovskites with bimolecular $\left(P L Q Y_{B i}\right)$ and excitonic $\left(P L Q Y_{E x}\right)$ recombination, respectively.

$$
\begin{gathered}
P L Q Y_{B i}=\frac{k_{2} \times n^{2}}{k_{\text {trap }} \times n+k_{2} \times n^{2}+k_{3 b i} \times n^{3}} \\
P L Q Y_{E x}=\frac{k_{1} \times n}{k_{\text {trap }} \times n+k_{1} \times n+k_{3 e x} \times n^{2}}
\end{gathered}
$$
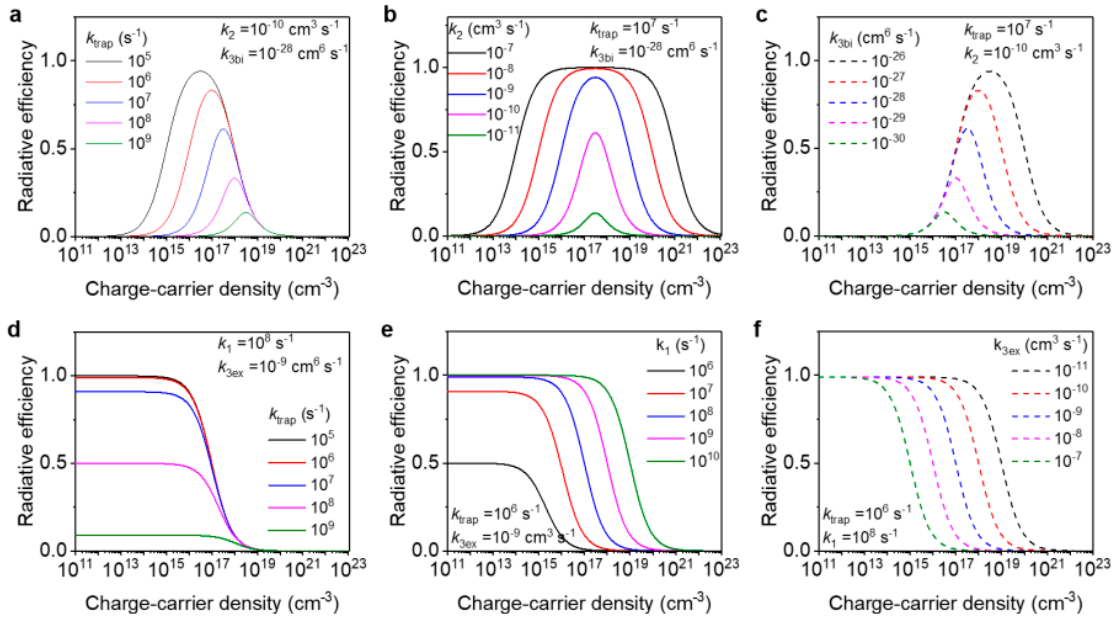

Figure 2.2 Charge-carrier recombination kinetics. Carrier density dependent PLQY of perovskites with $(\mathrm{a}-\mathrm{c})$ bimolecular recombination and $(\mathrm{d}-\mathrm{f})$ excitonic recombination. 
where $n$ is the charge-carrier density; $k_{\text {trap }}, k_{1}$, and $k_{2}$ are recombination coefficients of trap-assisted non-radiative recombination, excitonic radiative recombination, and bimolecular radiative recombination, respectively. $k_{3 b i}$ and $k_{3 e x}$ and three-body Auger recombination in the emissive materials with bimolecular recombination and excitonic recombination, respectively.

Figure 2.2 illustrates the dependence of the PLQYs on excitation carrier densities and different recombination coefficients in perovskites with bimolecular and excitonic recombination, respectively (The fixed recombination coefficients are extracted from Reference ${ }^{43}$ ). This figure indicates the importance of increasing excitonic and bimolecular recombination coefficients and decreasing trap-assisted and Auger nonradiative recombination coefficients. Thus, allowing the PLQYs to approach unity in a wide excitation density range relevant for practical LED operation.

\subsubsection{Reducing the Trap Density}

As discussed above, trap-assisted non-radiative recombination is a detrimental factor affecting the PLQYs in perovskites. At a low carrier density, deep level trap states have been determined to be the dominant

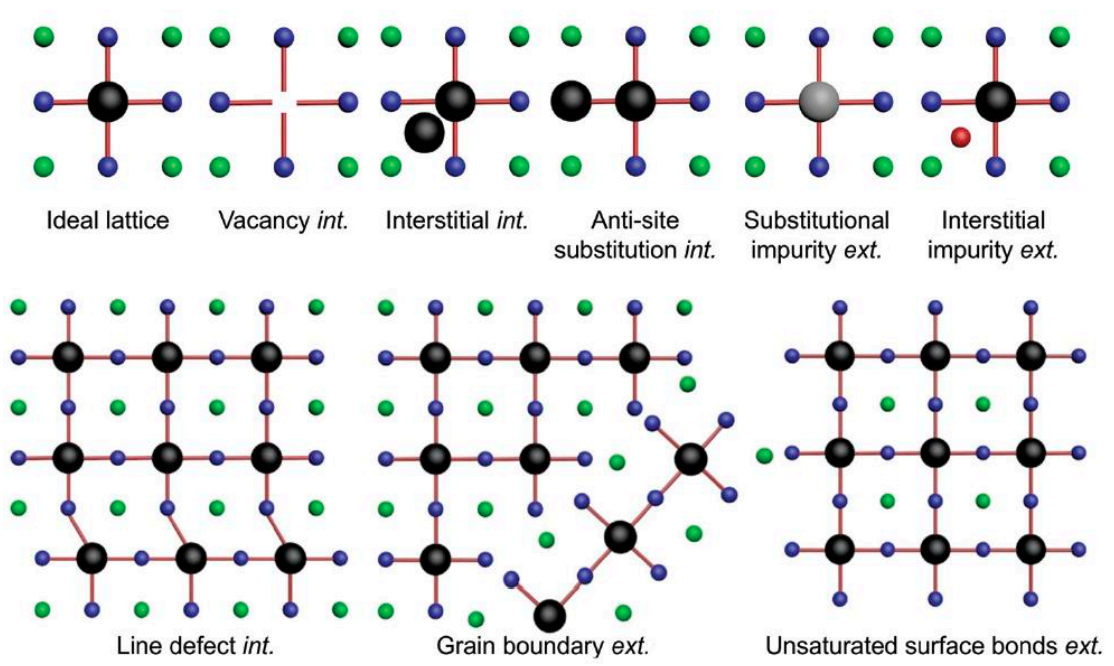

Figure 2.3 Schematic overview of intrinsic (int.) and extrinsic (ext.) defect types in perovskites, relative to an ideal lattice. ${ }^{44}$ 
pathway for the trap-assisted non-radiative recombination in perovskite films. ${ }^{44}$ As shown in Fig. 2.3, these trap states are usually associated with intrinsic point defects (e.g. vacancies, interstitials, and anti-site occupations), impurities, and extended defects (including line defect, grain boundaries, and surface defects). ${ }^{44}$ The trap density in perovskite films has been demonstrated to strongly depend on the employed (post-) processing procedures. Therefore, reducing trap density can be realized through controlling the growth process of perovskite film or defect passivation process.

Controlling the growth process of perovskite films is an important strategy to reduce the trap density, because the formation of defects during film growth process is governed by crystallization kinetics and dynamics. Various annealing processes, such as solvent/antisolvent engineering ${ }^{45-47}$, thermal annealing engineering ${ }^{48}$, and radiative thermal annealing, ${ }^{49}$ have shown significant effects on the crystallization process. Due to the inevitably induced vacancy defects during the crystallization process, a modest annealing temperature is supposed to reduce the vacancy defects. It requires the targeted high-quality perovskites a low formation energy and crystallization temperature. To realize high-quality perovskite films with high PLQYs at a modest annealing temperature, several approaches have been developed, including solvent-engineering approach to reduce the crystallization temperature, ${ }^{50}$ intermediate engineering to control the perovskite self-assembly crystallization, ${ }^{51}$ and excess organic halides or polymers loading in perovskite precursor solutions to reduce the perovskite formation energy. ${ }^{41,52,53}$ In addition, lead halide components have lower solubility in solvents (for instance dimethyl sulfoxide $\left(\left(\mathrm{CH}_{3}\right)_{2} \mathrm{SO}\right.$, DMSO $)$ and dimethylformamide $\left.\left(\mathrm{HCON}\left(\mathrm{CH}_{3}\right)_{2}, \mathrm{DMF}\right)\right)$ than perovskites. This induces the fast formation of Lewis adducts, such as $\mathrm{PbI}_{2} \cdot \mathrm{DMF}$ or $\mathrm{PbI}_{2} \cdot \mathrm{DMSO},{ }^{54,55}$ from perovskite precursor solutions prior to perovskite crystals under an uncontrolled solidification. The quickly formed Lewis adducts most probably lead to the formation of defects. Retarding the crystallization process has proved to be an effective approach to improve the quality of perovskite films with improved PLQYs ${ }^{56,57}$ Detailed information about controlling the growth of perovskite films can be found in Chapter 3 .

Defect passivation is another strategy to reduce trap density in perovskite films. By employing passivation agents in perovskite precursor solutions or via post-treatment on the crystallized perovskite films, the charged defects (vacancy defects and unsaturated surface bonds) 
are neutralized since they can provide extra coordination or ionic bonding, resulting in annihilation of electronic trap-states. ${ }^{58}$ Various passivation agents, such as Lewis acid/base molecules containing $-\mathrm{NH}_{2}$ or $-\mathrm{P}=\mathrm{O}$ groups, ${ }^{8,59}$ positively/negatively charged organic salts, ${ }^{60}$ and alkali metal ions ${ }^{61}$, have been investigated to reduce the trap density of perovskite films, resulting in enhanced PLQYs.

\subsubsection{Enhancing the Radiative Recombination}

Early-stage development of PeLEDs was based on three-dimensional (3D) bulk perovskite films, which have been widely studied in perovskite solar cells. However, 3D bulk perovskite films generally exhibit small exciton binding energies and possess bimolecular recombination with low recombination rates, limiting the radiative recombination efficiency. Confining the charge carriers in perovskite emitters is necessary to enhance radiative recombination and achieve high PLQY.

Spatial confinement of charge carriers is a useful strategy to improve the radiative recombination efficiency. The first room-temperature PeLEDs have demonstrated effective confinement of electrons and holes in a thin perovskite layer. ${ }^{7}$ Afterward, spatial confinement was also obtained by decreasing the size of perovskite grains, resulting in improved PLQYs of the prepared perovskite films and consequently enhanced EQEs of PeLEDs. ${ }^{62-65}$ In addition, controlling the cation alloying has been effective in confining one of the carriers through local variations in band edge energies and thus enhancing the PLQY. ${ }^{66}$

In addition to spatial confinement of free carriers to enhance bimolecular radiative recombination, changing the bimolecular radiative recombination to excitonic radiative recombination in perovskite films is another effective strategy to realize high PLQYs. Excitonic radiative recombination requires the size of perovskite crystals smaller than their Bohr diameters. One approach to reach this purpose is to synthesize colloidal perovskite nanocrystals (NCs). ${ }^{58}$ With rational optimization of compositions, structures, and sizes of perovskite NCs, the PLQYs of these materials have reached unity. ${ }^{67}$ Another approach is to form twodimensional (2D) and mixed-dimensional (including 2D and 3D perovskites) perovskites, which exhibit high exciton binding energies of up to hundreds of meV and high PLQYs. ${ }^{68-70}$ The emergence of 2D and mixed-dimensional perovskites, which will be discussed in Chapter 3, represents an important breakthrough in PeLEDs. 


\subsubsection{Suppressing the Three-Body Auger Recombination}

The three-body Auger recombination is a non-radiative process under high charge-carrier densities, where an electron-hole pair passes recombination energy to another charge carrier. Generally, the three-body Auger recombination can be one of the reasons for the efficiency roll-off at high current densities in LEDs, ${ }^{71-73}$ limiting the brightness of LEDs.

2D perovskite-based PeLEDs are typical example of this, which suffer from serious efficiency roll-off. Thus, it has been considered as the result of three-body Auger recombination due to high local carrier density. ${ }^{43}$ The serious efficiency roll-off in 2D perovskite-based PeLEDs can be assuaged by mixed-dimensional perovskite films, where 2D perovskite films are mixed with 3D components. The mixed-dimensional perovskites exhibit efficient charge transfer from low dimensional components to high dimensional components, beneficial to the achievement of high-brightness PeLEDs. ${ }^{73,74}$

\subsection{Optimizing the Injection Efficiency}

Charge-transport layers and interfacial layers have significant effects on injection efficiency. Optimizing charge-transport materials and interfacial layers is important for efficient and balanced carrier injection. In addition, perovskite layers exhibit strong physical/chemical interactions with charge-transport/interfacial layers. Proper chargetransport layers and interfacial layers are beneficial for suppressing nonradiative recombination at the interfaces of PeLEDs. As such, special attention should be paid to the charge transport layers and interfacial layers in PeLEDs.

\subsubsection{Underlying Charge-Transport Layers and Interfacial Layers}

The properties of the underlying layers can significantly affect the charge carrier injection as well as the growth process of perovskite films.

In a $p-i-n$ device structure, mixing PEDOT:PSS with a $\mathrm{MoO}_{3}$ ammonia solution or inducing a perfluorinated ionomer (PFI) thin layer between the PEDOT:PSS layer and perovskite layer can significantly enhance hole injection. ${ }^{75-77}$ Meanwhile, hydrophilic polymers, such as polyvinylpyrrolidone (PVP) and poly $\left(\left(9,9-\mathrm{bis}\left(3^{\prime}-(\mathrm{N}, \mathrm{N}-\right.\right.\right.$ dimethylamino)propyl)-2,7-fluorene)-alt-2,7-(9,9-dioctylfluorene)) (PFN), have been demonstrated to improve the interfacial contact 
between the hole transport layers and the perovskite layers. ${ }^{78,}{ }^{79}$ In addition, hole transport materials with suitable energy levels, such as $\mathrm{NiO}_{\mathrm{x}}$, exhibit effective hole transportation and electron blocking; meanwhile, they facilitate the formation of high-quality perovskite films. ${ }^{80}$

In a n-i-p device structure, a high-energy hydrophilic $\mathrm{ZnO}$ nanoparticle surface modified by multifunctional polyethyleneimine (PEI) layer or a hydrophilic insulating polymers (polyvinyl pyrrolidine, PVP) layer has demonstrated effective in promoting the formation of highquality perovskite films and balancing charge injection. ${ }^{81,} 82$ The underlying layers also affect the assembly of perovskite films, resulting in significant changes in morphology and electrical properties. ${ }^{83}$

These results not only reveal the critical roles of the underlying charge-transport/interfacial layers on carrier injection and growthprocess of perovskite films, but also call for special attention to the characterizations of perovskite films, such as single carrier devices, which are commonly used to evaluate charge injection/transport balance.

\subsubsection{Interfacial Layers on Top of Perovskite}

Loading an interfacial layer on top of prepared perovskite layers is another useful strategy to balance the carrier injection as well as passivate surface defects. For example, an insulating layer of poly (methyl methacrylate) (PMMA) has been inserted between the perovskite layer and the electron transport layer (ETL) to improve the balance of carrier injection. ${ }^{4}$ In addition, interfacial materials, including those with passivating moieties, are likely to induce additional healing of the surface defects. Trioctylphosphine oxide (TOPO) ${ }^{59}$ and amine materials (e.g. 2,2' -(ethylenedioxy)diethylamine $(\text { EDEA })^{8}$ and a mixture of ethylenediamine (EDA) and polyethylenimine $(\mathrm{PEI})^{84}$ ) have been demonstrated to show passivation effects on perovskite layers. Therefore, passivation interlayers with different functional groups deserve further investigations.

\subsection{Improving the Light Outcoupling Efficiency}

In addition to the rational design of the emissive layers, interfacial layers, and charge-transport layers, enhancing the light outcoupling efficiency is also important for further improvement of the device efficiencies. Optical 
simulations based on planar-structured devices without any light extraction technologies reveal the critical roles of the thickness of perovskite layers, and predict $25 \%$ and $20 \%$ outcoupling efficiency $\left(\eta_{o p}\right)$ for PeLEDs based on 3D perovskites and mixed-dimensional perovskites, respectively. ${ }^{85}{ }^{86} \mathrm{In}$ addition, light extraction technologies, such as substituting the flat substrate with a nanophotonic substrate or using nanoimprinted moth-eye nanostructures combined with a half-ball lens, have been demonstrated to enhance the light outcoupling efficiencies of PeLEDs. $^{42,87}$

Different from traditional light extraction approaches, modulating the morphologies of perovskite films provides unique opportunities to improve the light outcoupling efficiencies of PeLEDs. This approach not only avoids the use of complicated manufacturing processes, but also retains the wavelength- and viewing-angle-independent electroluminescence. Perovskite emissive layers consisting of isolated sub-micrometre-sized platelets have been demonstrated with enhanced light outcoupling efficiency approaching 30\%, leading to PeLEDs with a high peak EQE of $20.7 \% .{ }^{5}$ Meanwhile, the discontinuous films do not necessarily induce the electrical issue, such as the leakage current/electrical shunts. ${ }^{5,8}, 83$ In addition, anisotropic photon emission in perovskite NCs suggests that high light outcoupling efficiencies in PeLEDs could be obtained by using perovskites with in-plane transition dipole moments. ${ }^{88,89}$ 


\section{Chapter 3 High-Quality Perovskite Films}

Metal halide perovskites have rapidly emerged as a hot topic due to their excellent optoelectronic properties, such as high photoluminescence quantum yields (PLQYs), widely tunable bandgap, narrow emission, and high charge carrier mobility. ${ }^{90}$ They have been investigated in a broad range of applications, including solar cells (PSCs), light-emitting diodes (PeLEDs), lasers, photodetectors, field-effect transistors, and resistiveswitching memory devices. ${ }^{90-92}$ Among these applications, PSCs and PeLEDs are most studied research subfields and show rapid progress in efficiency; both the power conversion efficiency (PCE) of PSCs and external quantum efficiency (EQE) of PeLEDs are over $20 \%$ within several years. ${ }^{4,5,83}$ For PSCs and PeLEDs, high-quality perovskite films are regarded as a fundamental requirement towards devices with highefficiency and long-term operational stability.

In PSCs, various strategies have been developed for achieving highquality perovskite films. ${ }^{69}, 94,95$ Some strategies have been imitated in PeLEDs, such as loading additives in the perovskite precursor solutions and employing mixed-dimensional perovskites ${ }^{5,8}$. However, due to different working mechanisms, PeLEDs have some different requirements from PSCs regarding high-quality perovskite films. For example: 1. To achieve high-performance PSCs, large perovskite grains are required for effective light absorption and charge carrier transport and extraction. ${ }^{94}$ However, small perovskite grains are preferred for PeLEDs because of the requirement of effective confinement of electron-hole pairs. $^{7}$ 2. Mixed-dimensional perovskite films reduce the photocurrents of PSCs due to the relatively poor charge transport of the lowdimensional components. ${ }^{96,97}$ However, PeLEDs show high tolerance to the charge transport, and PeLEDs based on mixed-dimensional perovskite films with high two-/three-dimensional (2D/3D) ratios have frequently demonstrated high performance. ${ }^{41}$ Therefore, in spite of rich experience in PSCs, strong efforts are still desired to achieve high-quality perovskite films towards high-efficiency and stable PeLEDs.

The deposition of high-quality perovskite films generally includes a series of complex processes, depending on processing conditions and specific material systems. In this chapter, we outline the key challenges lying in preparation of high-quality perovskite films for highperformance PeLEDs, and discuss several effective synthetic strategies to 
tackle these challenges, including composition engineering, additive engineering, modulation of growth process, and post-treatment of crystallized perovskite films.

\subsection{Challenges}

As discussed in Chapter 2, perovskite films with effective charge confinement and low defects are essential to achieve efficient and stable PeLEDs. However, perovskite films deposited from stoichiometric perovskite precursor solutions through spin-coating face some challenges. ${ }^{94}$ For example, fast crystallization of components from the perovskite precursor solution induces polycrystalline perovskite films with large perovskite grain sizes; insufficient reaction among various components and/or partial decomposition of components result in undesired components; incomplete phase transformation causes undesired phases.

\subsubsection{Large Perovskite Grain Sizes}

The crystallization process is an essential process for the formation of crystalline films, dictating the characteristics of the final film. The crystallization process consists of two major events, that is, nucleation and crystal growth. ${ }^{98}$ Nucleation is the first step of crystallization. It occurs when the concentration of solutes reaches saturation, resulting in the appearance of crystalline particles. The crystal growth process is the increase in the size of the particles and leads to a crystal state. However, multiple ions and polar aprotic solvents in perovskite precursor solutions make the crystallization process complicated.

Generally, the perovskite precursor solution is prepared by mixing monovalent cation halide $\left(\mathrm{AX}, \mathrm{A}=\mathrm{MA}^{+}, \mathrm{FA}^{+}\right.$, or $\mathrm{Cs}^{+}, \mathrm{X}=\mathrm{I}^{-}, \mathrm{Br}^{-}$, or $\left.\mathrm{Cl}^{-}\right)$ and lead halide $\left(\mathrm{PbX}_{2}\right)$ with a stoichiometric $(1: 1)$ molar ratio in a polar solvent (e.g. dimethyl sulfoxide $\left(\left(\mathrm{CH}_{3}\right)_{2} \mathrm{SO}\right.$, DMSO) and dimethylformamide $\left.\left(\mathrm{HCON}\left(\mathrm{CH}_{3}\right)_{2}, \mathrm{DMF}\right)\right)$. Due to the strong coordination between the oxygen atoms of the solvents and the $\mathrm{Pb}$ atoms, the perovskite precursor solution is not seriously considered as a "true" solution; instead, it includes large colloidal particles (approximately $\mu \mathrm{m}$ in size) with one-dimensional (1D) $\mathrm{PbX}_{2} \bullet \mathrm{DMF}\left(\mathrm{PbX}_{2} \bullet \mathrm{DMSO}\right)$ Lewis adducts along (001) plane as shown in Fig. 3.1.54, 94, 99, 100 The low solubility of lead halide components and the high boiling point of the 


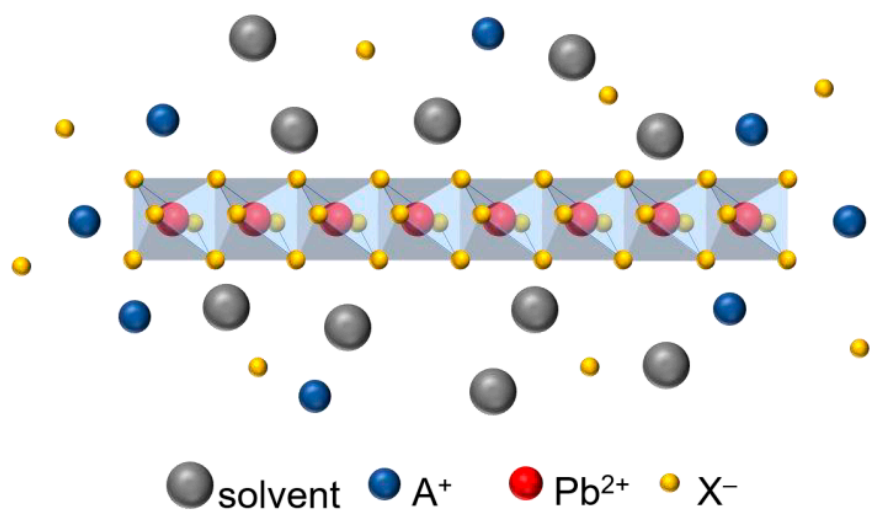

Figure 3.1 Diagram of $\mathrm{Pb}$-based Lewis adducts in perovskite precursor solution.

solvent lead to fast precipitation of the large-sized lead halide Lewis adducts from the perovskite precursor solution prior to the crystallization of perovskite nuclei. Subsequently, A-site cations (e.g. $\mathrm{MA}^{+}$cations) quickly interact with the $\mathrm{PbI}_{2}$ from the (001) plane edges, resulting in a nanorod micro-morphology. ${ }^{101}$ The fast crystallization process also leads to randomly oriented crystal planes and numerous defects. ${ }^{53,60,102}$

\subsubsection{Undesired Components}

In addition to the large grain size of perovskite films, the prepared perovskite films suffer from undesired components, which can be nonradiative recombination channels.

During the growth process of perovskite film, wet perovskite films (after spin-coating and before thermal annealing) generally emerge as intermediates (e.g. quasi-ternary complexes of $\mathrm{MAI} \cdot \mathrm{PbI}_{2} \cdot \mathrm{DMF}$ or $\left.\mathrm{MAI} \cdot \mathrm{PbI}_{2} \cdot \mathrm{DMSO}\right) .{ }^{103}$ The monovalent cation halide is probably not fully coordinated with $\mathrm{PbI}_{2}$, leading to residual $\mathrm{PbI}_{2}$ in the perovskite films. ${ }^{54}$ In addition, due to the low decomposition temperature or reacted with sublayer, the prepared perovskites suffer from decomposition during the thermal annealing process, resulting in the formation of organic gases (e.g. $\left.\mathrm{CH}\left(\mathrm{NH}_{2}\right)_{2}, \mathrm{CH}_{3} \mathrm{NH}_{2}\right)$ and hydroiodic acid (HI) ${ }^{83,}, 104$ Therefore, as shown in Fig. 3.2a and 3.2b, both insufficient reaction among of components and decomposition of perovskites lead to undesired $\mathrm{PbI}_{2}$ and vacancy defects in the prepared perovskite film. ${ }^{51,105}$ 

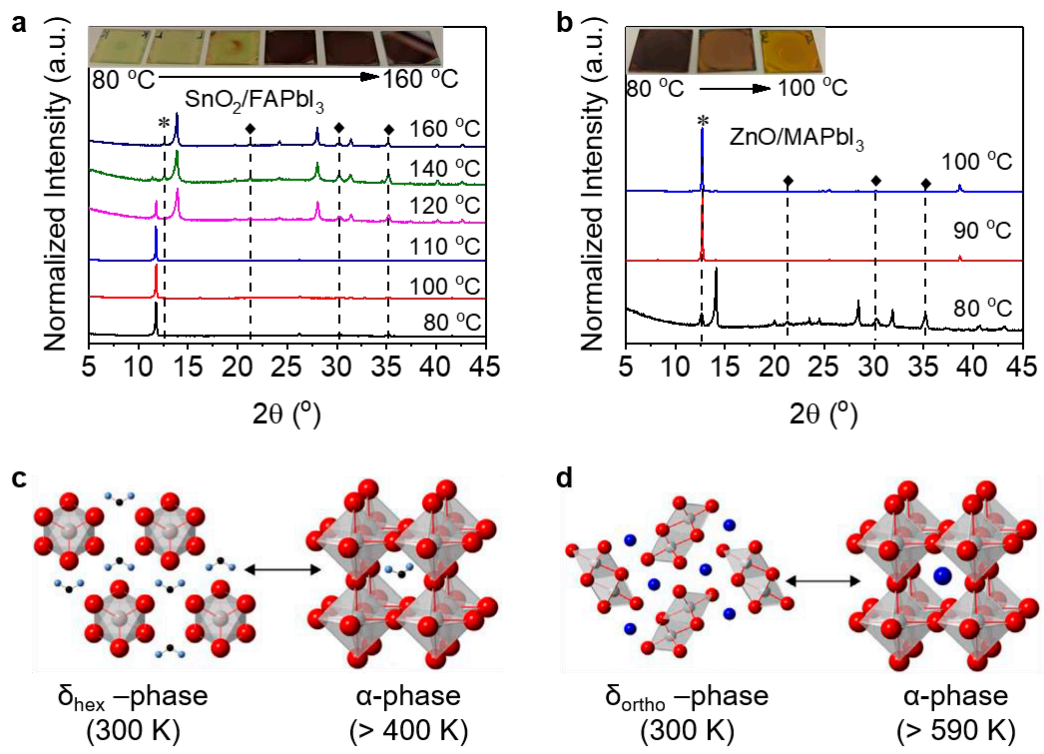

$\mathrm{FAPbl}_{3}$

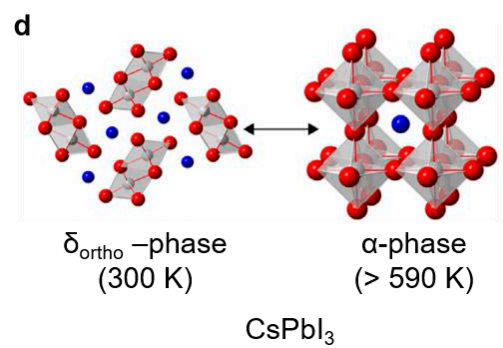

Figure 3.2 XRD patterns and photographs of (a) $\mathrm{FAPbI}_{3}$ and (b) $\mathrm{MAPbI}_{3}$ perovskite films. Thermo dynamically preferred room and high temperature crystal structure models for (c) $\mathrm{FAPbI}_{3}$ and (d) $\mathrm{CsPbI}_{3} .{ }^{107}$

Non-perovskite phase is another challenge for high-quality perovskite films. $\mathrm{FA}^{+}$and $\mathrm{Cs}^{+}$cations show stronger affinity than solvents (DMF and DMSO) toward $\mathrm{PbI}_{2},{ }^{51,106}$ avoiding the formation of the solvent-relevant intermediate phases. However, FA- and Cs-based perovskite possess more than one crystal structures as shown in Fig. 3.2c and 3.2d. ${ }^{107}$ For example, limited by the tolerance factor, the cubic structures of $\mathrm{FAPbI}_{3}$ and $\mathrm{CsPbI}_{3}$ perovskites are not stable at room temperature. In contrast, the non-perovskite structures (yellow phases) are stable structures at room temperature. The wet perovskite films, prepared from stoichiometric perovskite precursor solutions, exhibit yellow phases because they have low formation energies. In order to achieve the desired black phases, high annealing temperatures (over $160{ }^{\circ} \mathrm{C}$ and $300{ }^{\circ} \mathrm{C}$ for $\mathrm{FAPbI}_{3}$ and $\mathrm{CsPbI}_{3}$, respectively) are generally required for the transformation from yellow phases to black phases. The black-phase perovskite films are thermodynamically unstable and will transfer into yellow phases at room temperature, exhibiting poor long-term stability. 


\subsection{Composition Engineering}

Vacancy defects and undesired components in perovskite films have been considered as the predominant sources of charge trapping for limiting the efficiency of PeLEDs. ${ }^{8,} 67,108$ To solve these issues, composition engineering, including non-stoichiometric perovskite precursors and mixed-dimensional perovskites, can be a useful strategy.

\subsubsection{Non-Stoichiometric Perovskite Precursors}

Metal halide perovskites, with the general formula of $\mathrm{ABX}_{3}$, require a stoichiometric (1:1) reaction between $\mathrm{AX}$ and $\mathrm{BX}_{2}$ components. However, the non-fully coordination between monovalent cation salts and lead halides and/or the decomposition of perovskite lead to the formation of vacancy defects and/or $\mathrm{PbX}_{2}$ in perovskite films. ${ }^{54}$ Hence, excess $\mathrm{AX}$ components in perovskite precursors are developed to provide an AXrich condition, resulting in a range of benefits.

Adequate AX salts in the perovskite precursor solution can promote the transformation of $\mathrm{PbX}_{2}$ into perovskite phase. For example, adequate MAI in the perovskite precursor solution can retard the crystallization of 1D $\mathrm{PbI}_{2}$ chain and shift the low coordinated $\mathrm{PbI}_{2}$ to high coordinated plumbate species $\left(\mathrm{PbI}_{3}{ }^{-}, \mathrm{PbI}_{4}{ }^{2-}, \mathrm{PbI}_{5}{ }^{3-}\right.$, and $\left.\mathrm{PbI}_{6}{ }^{4-}\right) .{ }^{54,101,109}$ Meanwhile, the colloid sizes in the perovskite precursor solution were decreased. The prepared perovskite films from the perovskite precursor solutions with excess AX exhibit small perovskite grain sizes and no $\mathrm{PbI}_{2}$ residue. ${ }^{63,83 \text {, }}$ $110-112$

Excess AX in perovskite precursors are also of benefit as they reduce vacancy defects. Excess AX salts not only provide an AX-rich condition for the full reaction with $\mathrm{PbX}_{2}$, but also compensate the possible volatilization of organic $\mathrm{AX}$, such as MAI, MABr, and FAI. Meanwhile, excess AX may aggregate around perovskite grains to passivate defects on the grain surfaces or boundaries, resulting in improved EQEs of PeLEDs. ${ }^{4,53,83,113}$ In addition, excess monovalent cation salts (e.g. FAI) in perovskite precursor can decrease the formation energy of black phase $\mathrm{FAPbI}_{3}$ perovskite and decrease the thermal annealing temperature to 100 ${ }^{\circ} \mathrm{C}$, owing to the formation of wide-bandgap intermediate phase. ${ }^{53}$ Low formation energy and low thermal annealing temperature decrease the number of vacancy defects.

In addition, excess $\mathrm{AX}$ in perovskite precursors maybe induce the formation of low dimensional perovskite films. For example, a large 
molar ratio of $\mathrm{MABr}$ in the perovskite precursor solution leads to the insitu formation of $\mathrm{MAPbBr}_{3}$ nanocrystals. Meanwhile, the $\mathrm{MAPbBr}_{3}$ nanocrystals were embedded in the unreacted wider-bandgap $\mathrm{MABr} .{ }^{111}$ Excess FAI in the $\mathrm{FAPbI}_{3}$ precursor solution can result in mixeddimensional perovskite films, observed by absorption and PL spectra, on $\mathrm{SnO}_{2}$ (or $\mathrm{TiO}_{2}$ ) substrates. ${ }^{83}$

\subsubsection{Large-Sized Organic Ammonium Salts}

In addition to loading rational amounts of excess AX (e.g. MAX, FAX, and $\mathrm{CsX}$ ) in perovskite precursor solutions, large-sized organic monoammonium or diammonium salts with a general formula of $\mathrm{R}$ $\mathrm{CH}_{2} \mathrm{NH}_{3} \mathrm{X}$ or $\mathrm{R}-\left(\mathrm{CH}_{2} \mathrm{NH}_{3} \mathrm{X}\right)_{2}$, respectively, have been developed to partially or fully replace AX in perovskite precursor solutions for efficient PeLEDs. Due to their large sizes, these ammonium cations cannot maintain the $\mathrm{ABX}_{3}$ crystal structure by taking the position of the A-site cations; instead, they will split the $\mathrm{ABX}_{3}$ structure into low-dimensional structures, leading to the formation of 2D, quasi-2D perovskites or mixeddimensional perovskites (the mixtures of 2D, Q-2D and 3D perovskites). As shown in Fig. 3.3a, 2D/quasi-2D perovskites share a formula of $L_{2} A_{n-}$ ${ }_{1} \mathrm{~B}_{\mathrm{n}} \mathrm{X}_{3 \mathrm{n}+1}$ (Ruddlesden-Popper (RP) structure) or $\mathrm{LA}_{\mathrm{n}-1} \mathrm{~B}_{\mathrm{n}} \mathrm{X}_{3 \mathrm{n}+1}$ (DionJacobson (DJ) structure), depending on the monoammonium and diammonium salts, respectively. Here $n$ represents the number of inorganic octahedra sheets between the large-sized organic spacers. In addition, amine derivatives loaded into the precursor are also able to form mixed-dimensional perovskites due to the in-situ protonation process of the large-sized amine (Paper 2). Meanwhile, the remaining amine can passivate vacancy defects. ${ }^{114,115}$ Considering various combinations of 3D perovskites and abundant large-sized organic spacers, a wide range of options have been developed for efficient PeLEDs.

Compared with $3 \mathrm{D}$ perovskites, the $\mathrm{PbX}_{6}{ }^{4-}$ frameworks in mixeddimensional perovskites are separated by two large-sized organic layers, as shown in Fig. 3.3b, giving rise to multiple-quantum-well structures. The inorganic slabs serve as potential "wells", while the large-sized organic layers function as potential "barriers". Although large-sized organic spacers act as potential "barriers", the mixed-dimensional perovskites exhibit energy transfer from small-n perovskite components to large-n perovskite components, resulting in high local carrier densities. The photoluminescence (PL) emission is mainly from the large-n 


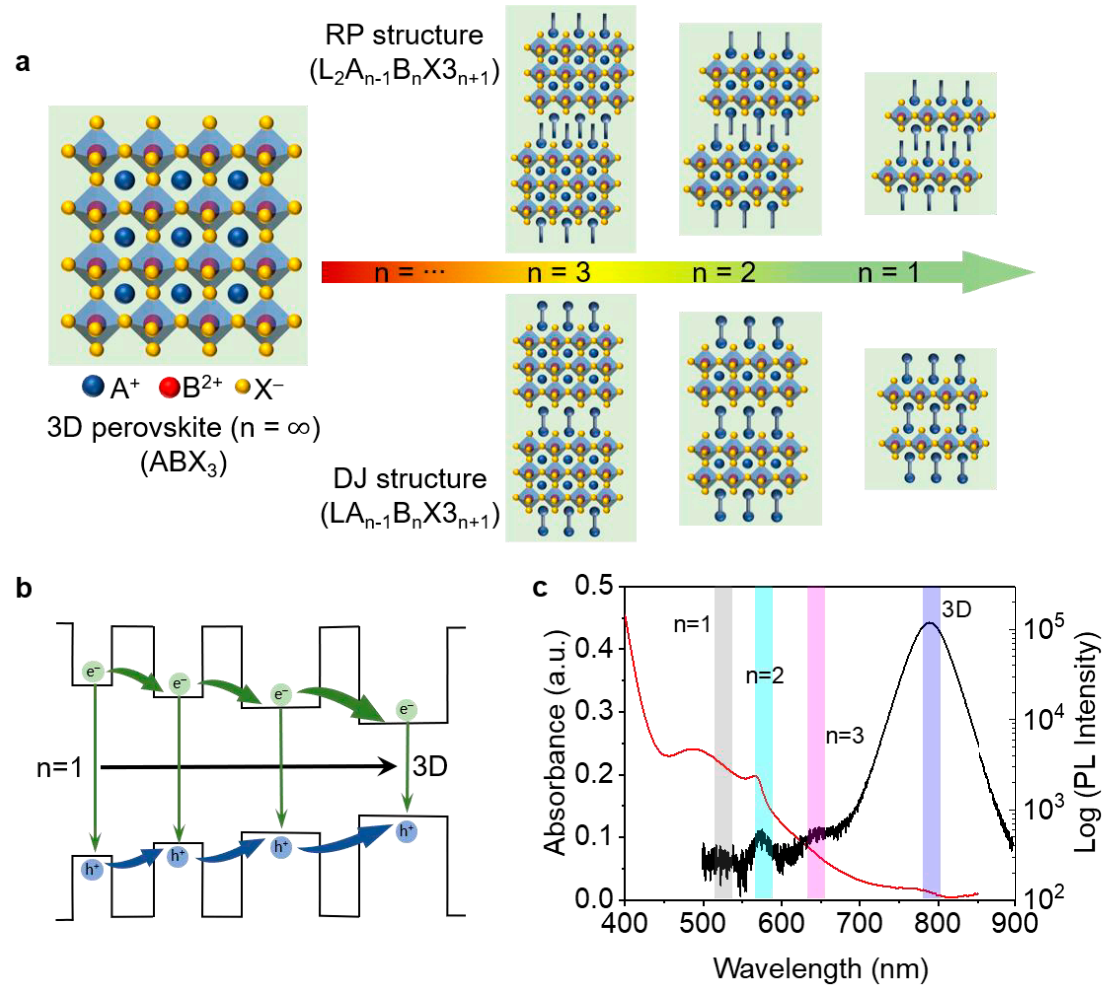

Figure 3.3 The structures and optical properties of mixed dimensional perovskites. (a) Diagram of 3D, 2D ( $\mathrm{n}=1)$, and quasi-2D perovskites. (b) Diagram of charge-carrier transfer and recombination in mixed dimensional perovskites. (c) The absorption and PL spectra of a mixed dimensional perovskite thin film (Paper 4).

perovskite components with weak emission from the small-n perovskite components. Optimizing the ratio between AX and large-sized organic spacer in perovskite precursor solutions (Paper 3) and choosing rational deposition methods (Paper 4) can lead to perovskite films with various $n$ values (Fig. 3.3c).

In addition, reducing the dimensionality of the perovskites also increases their exciton binding energy (hundreds of meV), which can be effectively tuned through a careful control of the thickness of the inorganic wells (the $\mathrm{n}$ values). ${ }^{69,70}$ Moreover, the recombination model can change from bimolecular radiative recombination (3D perovskite) to excitonic (2D/quasi-2D perovskites) radiative recombination by reducing 
the dimensionality of the perovskites. ${ }^{43}$ For mixed-dimensional perovskites, both recombination models may exist since they have various $2 \mathrm{D}$ /quasi-2D phases as well as $3 \mathrm{D}$ phases.

\subsection{Additive Engineering}

Benefiting by composition engineering, the quality of perovskite films can be enhanced. ${ }^{4,73}$ However, LEDs based on these kinds of perovskite films still deliver modest efficiency and stability. Additives have been employed in the perovskite precursor solutions to further improve the quality of perovskite films.

\subsubsection{Additive-Assisted Crystallization}

Generally, the crystallization processes of perovskites can be strongly mediated by additives in the perovskite precursor solutions. Proper selection of additives can play a critical role in the deposition of highquality perovskite films. Although the mechanism of the additive-assisted crystallization process remains unclear, researchers have proposed some possible explanations.

Here, we take additives with the amino group $\left(-\mathrm{NH}_{2}\right)$ as an example since the amino group is a popular functional group widely used for achieving high-quality perovskite films. ${ }^{5,8,56}$ As shown in Fig. 3.4a-c, the use of 4,4'-diaminodiphenyl sulfone (DDS) in the perovskite precursor solution leads to enhanced crystallinity and modified morphology of perovskite films. The alkylamino group was normally considered as Lewis bases, which can form hydrogen bonds with $\mathrm{FA}^{+}$cation through the electron-donating ability of the amino groups and subsequently affect the crystallization..$^{5}$ The hydrogen-bonding ability between amino group and $\mathrm{FA}^{+}$cation can be affected by the chain length ${ }^{5}$ or the electronwithdrawing group in the additive ${ }^{8}$. However, when the additive includes a strong electron-withdrawing group (e.g. $-\left(\mathrm{C}_{6} \mathrm{H}_{4}\right) \mathrm{SO}_{2}\left(\mathrm{C}_{6} \mathrm{H}_{4}\right)-$ group, as shown in Fig. 3.4a. Paper 1), the amino groups will show electropositivity and form hydrogen bonds with the $\mathrm{I}^{-}$anions rather than the $\mathrm{FA}^{+}$cations, consequently modifying the perovskite crystallization process to realize high-quality perovskite films. Fig. $3.4 \mathrm{~d}$ is a schematic illustration that shows the growth mechanism of perovskite films modified by DDS. Due to the weak coordination bonds between DDS and $\mathrm{I}^{-}$anion, it is easy for 
$\mathrm{FA}^{+}$cations to interact with the perovskite nuclei to form 3D perovskite films even in the condition of a high concentration of DDS in the perovskite precursor solutions (10 times molar ratio of DDS to perovskites). In addition, DDS wrapping at the surface of perovskite crystals could protect these crystals, leading to their long-term stability in ambient conditions.

In addition to the additives with amino groups, other additives can also assist the growth process of perovskite films. For example, the additive polyethyleneglycol (PEG) promotes the formation of highly compact and pinhole-free $\mathrm{CsPBr}_{3}$ perovskite films. ${ }^{116}$ Organic molecule, 1,4,7,10,13,16-hexaoxacyclooctadecane, suppresses the crystallization of the phenethylammonium bromide $(\mathrm{PEABr})$ phase and improves the domain size distribution in the $\mathrm{PEA} / \mathrm{CsPbBr}_{3}$ mixed-dimensional perovskite film. ${ }^{117}$

a

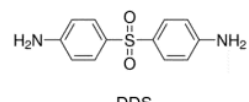

c

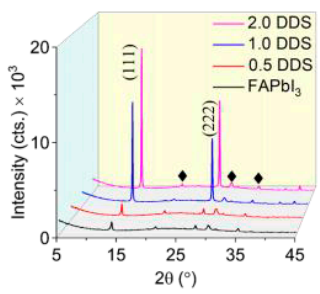

d

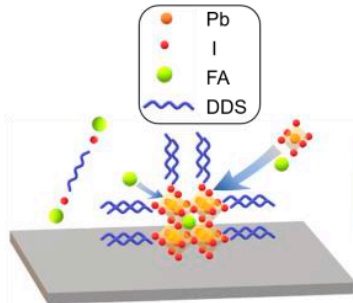

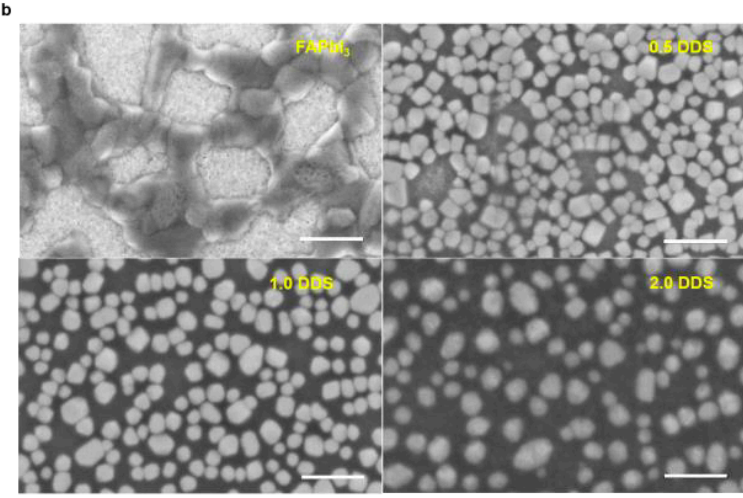

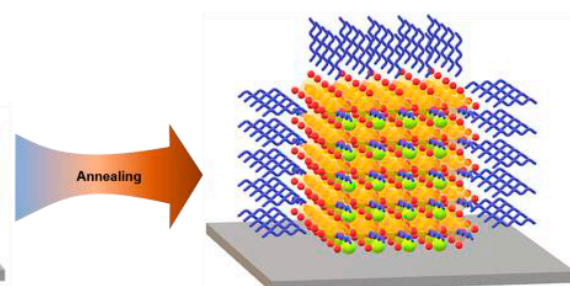

Figure 3.4 Additive-assisted crystallization process (Paper 1). (a) Molecular structure of additive (DDS, in Paper 1). (b) top-view SEM images of perovskite films with various amount of additive and (c) XRD patterns of perovskite films. (d) Schematic illustration of the additive-assisted growth mechanism. 


\subsubsection{Defect Passivation}

In-situ defect passivation during the deposition of perovskite films is another benefit of additives in perovskite precursor solutions. The ionic defects, such as halide vacancies, have been considered as the main trap states for the limited EQE of PeLEDs. ${ }^{8}$ To neutralize the halide vacancy defects, molecules or polymers containing one or more Lewis base moieties (such as $-\mathrm{NH}_{2},-\mathrm{COOH},-\mathrm{OH}$, and $-\mathrm{P}=\mathrm{O}$ ) ${ }^{60,118,119}$ have been demonstrated to reduce the defect densities through Lewis acid-base interaction.

Besides Lewis bases, electron-transport small molecules, such as 2,2',2"-(1,3,5-benzinetriyl)-tris(1-phenyl-1-H-benzimidazole) (TPBi) and 1,3,5-tri(m-pyridin-3-ylphenyl)benzene (TmPyPB), were also used as additives in perovskite precursor solutions. ${ }^{120,121}$ They not only transport carriers, but also reduce the defect densities on the grain boundaries and modify the morphology of perovskite films.

\subsection{Modulation of Growth Process}

One-step spin-coating method (Fig. 3.5a) is the most used deposition method for high-efficiency PeLEDs, where the perovskite precursor solution is dropped onto the substrate before or during the spinning of the substrate. Generally, the growth process of the one-step spin-coating method can be divided into two steps: the spin-coating process and the thermal annealing process. During the spinning of substrates, most of the perovskite precursor solutions are removed by centrifugal force, resulting in a wet film. Afterwards, the remaining solvent evaporates from the wet film during thermal annealing, resulting in the formation of perovskite films. However, the evaporation of solvent is usually slow, during which the organic cations in organic-inorganic hybrid perovskites may suffer from volatilization.

To fast remove the solvent, an anti-solvent method was widely used in high-efficiency PSCs. However, the traditional anti-solvent method (such as dropping pure chlorobenzene or chloroform solution on spinning perovskite films) normally leads to large perovskite grains, which impede the carrier recombination in PeLEDs. To overcome this issue, an additivebased nanocrystal pinning $(\mathrm{A}-\mathrm{NCP})^{63,121,122}$ technique was developed as shown in Fig. 3.5b, where the chloroform/TPBi (or chloroform/SPPO1, 9,9-spirobifluoren-2-yl-diphenylphosphine oxide) mixture solution 


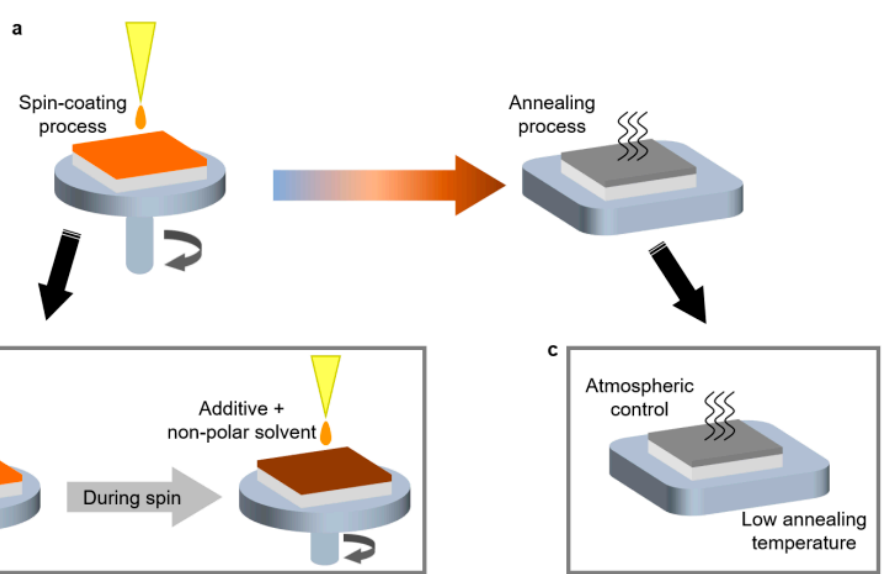

Figure 3.5 Perovskite film growth-process. (a) Traditional one-step spincoating method. (b) Modified anti-solvent method. (c) Annealing process engineering.

replaces the pure chloroform solution as the anti-solvent, realizing perovskite films with small grain size and low trap densities.

The formation of defects during the thermal annealing process is governed by the crystallization kinetics and dynamics. Therefore, thermal annealing treatment of perovskite films, including thermal annealing atmosphere and temperature as shown in Fig. 3.5c, is also important for reducing trap density. Low annealing temperature has proved to be beneficial to realize efficient and stable PeLEDs. ${ }^{48}$ In addition, light exposure, oxygen, atmospheric humidity, and pressure have been demonstrated to play significant roles in the perovskite crystallization process and the device performance of PSCs. ${ }^{44,}{ }^{123-126}$ Therefore, further investigations on the annealing process are desired to further improve the efficiency and stability of PeLEDs.

\subsection{Post-Treatment}

As discussed above, vacancy defects are hardly totally avoided during the crystallization process. To further reduce the surface vacancy defects, the deposition of rational passivation materials (dissolved in a non-polar solvent) on prepared perovskite films is another useful strategy that does not affect the crystallization and morphology of the perovskite films. For example, the passivation materials, such as tetraethylammonium bromide 
(TEABr) and amine derivatives, were deposited on the perovskite films to passivate the upper surface halide vacancy defects, resulting in enhanced PLQY of perovskite films and improved efficiency and stability of PeLEDs. ${ }^{58,59,127}$

Recrystallization through post-treatment is also investigated to achieve high-quality perovskite films. For example, the instant exposure of deposited $\mathrm{MAPbI}_{3}$ perovskite film in the methylamine (MA) gas atmosphere can form a liquid $\mathrm{MAPbI}_{3} \bullet \mathrm{xA}$ intermediate phase, which undergoes regrowth of $\mathrm{MAPbI}_{3}$ by degassing $\mathrm{MA}$ in an ambient environment. The prepared perovskite film exhibits improved crystallinity and PL intensity, resulting in the enhanced EQEs of PeLEDs. ${ }^{80}$ 


\section{Chapter 4 Ion Migration}

Ion migration, the movement of ions in materials, has been regarded as one of the most mysterious processes in perovskite light-emitting diodes (PeLEDs). On one hand, halide anions and monovalent cations moving to the surfaces or grain boundaries of perovskite films can passivate the surface vacancy defects, leading to improved photoluminescence (PL) intensity of perovskite films and enhanced external quantum efficiency (EQE) of PeLEDs. ${ }^{128,129}$ On the other hand, ion migration contributes to the unusual phenomena (e.g. hysteresis and scan model-dependent EQE) and the poor stability of PeLEDs. ${ }^{130}$ Therefore, understanding and regulating ion migration in metal halide perovskite materials are necessary for achieving efficient and stable PeLEDs. In this chapter, we discuss the roles of ion migration in PeLEDs. We start with an introduction of the origin and evidence of ion migration in perovskite materials. We then discuss the influence of ion migration on the efficiency and stability of PeLEDs. Finally, we summarize some promising approaches that can suppress the ion migration process.

\subsection{Ion Migration in Perovskites}

The investigation of ion migration phenomenon in perovskite-type materials can be dated back to the 1980s. Some perovskite oxides (e.g. $\mathrm{LaMnO}_{3}$ and $\mathrm{SrTiO}_{3}$ ) can even be regarded as ionic conductors. ${ }^{131,132}$ As for the metal halide perovskite materials, they are regarded as mixed electronic and ionic conductors. ${ }^{133}$ Meanwhile, ion migration in devices based on metal halide perovskites is considered as a general and intrinsic property, which is independent from the composition of perovskite materials and the device architectures. ${ }^{134,135}$

\subsubsection{Ion Migration Species}

Three-dimensional (3D) metal halide perovskites, with a general formula of $\mathrm{ABX}_{3}$, include three constituent ions (monovalent cation $\left(\mathrm{A}^{+}\right)$, halide anion $\left(\mathrm{X}^{-}\right)$and divalent metal $\left.\left(\mathrm{B}^{2+}\right)\right)$. In principle, all of them may migrate in the perovskite crystals or films. The activation energy $\left(E_{A}\right)$ is used to characterize the ability of ion movement, which is related to the crystal 
structure, the ion-jumping distance, the ionic radius, and the charge of ions. ${ }^{135}$ From both theoretical and experimental perspectives, the $\mathrm{A}^{+}$ cations and $\mathrm{X}^{-}$anions possess lower $E_{A}$ values than $\mathrm{B}^{2+}$ divalent metal cations. For example, for $\mathrm{MAPbI}_{3}$, the $E_{A}$ values of $\mathrm{I}^{-}$and $\mathrm{MA}^{+}$are calculated to be 0.58 and $0.84 \mathrm{eV}$, respectively, while the $E_{A}$ value of $\mathrm{Pb}^{2+}$ is up to $2.31 \mathrm{eV} .{ }^{136}$ Other theoretical results have shown a consistent tendency though with different $E_{A}$ values. ${ }^{137-139}$ The $E_{A}$ values of metal halide perovskite films were also experimentally estimated from the change of conductivity at different temperatures. ${ }^{140,141}$ The measured $E_{A}$ values are somewhat smaller than those predicted from the theoretical simulations. The inconsistency may be caused by the moving scenario. The theoretical values are calculated from a single crystal model with the ion migration in a bulk crystal through Schottky defects or Frenkel defects. In contrast, the experimentally measured $E_{A}$ values are complex results, which may be related to the mixed migration ions (both cations and anions) and the mixed migration channels (bulk defects, surfaces, and
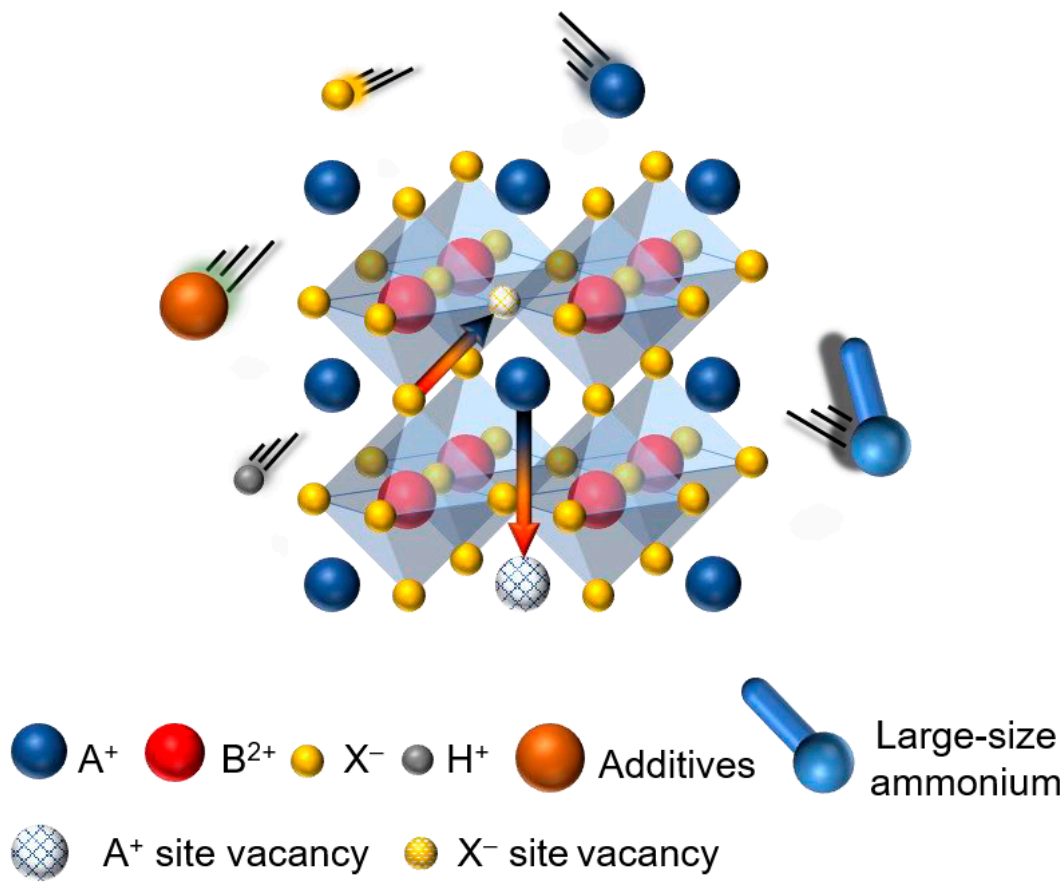

Figure 4.1 Mobile ion species in perovskites. 
grain boundaries). Both the theoretical and experimental results support that the $\mathrm{A}^{+}$cations and $\mathrm{X}^{-}$anions are the majority of mobile ions in metal halide perovskite films.

To reach high-efficiency PeLEDs, as discussed in Chapter 3, additional components are usually loaded in perovskite precursor solutions. Those components most probably remain in the perovskite films. Therefore, besides the mobile ions from the perovskite crystal structure, additional species (as shown in Fig. 4.1) also deserve consideration for the possible ion migration scenarios. For example, $\mathrm{H}^{+}$ has been theoretically speculated to migrate through Frenkel defects and shown a low $E_{A}$ of $0.29 \mathrm{eV}$ along a transient hydrogen bond connecting two equatorial iodides. ${ }^{142}$ In addition, large-sized organic ammonium, various additives, and non-stoichiometric precursors with excess $\mathrm{AX}$ components (Paper 5) can be mobile ions or exacerbate ion migration because these components in the perovskite films may significantly open up the crystal structure. ${ }^{135,143}$

\subsubsection{Ion Migration Channels}

Mobile ions migrate through special channels in perovskites under the electric field (the applied external electric field or the internal electric field generated by the photoelectric effects). Understanding the migration channels is of great importance for achieving high-efficiency and stable PeLEDs.

Theoretical results of first-principle calculations have already pointed out that bulk defects, including Schottky defects and Frenkel defects, are the main ion migration channels. For example, in the $\mathrm{MAPbI}_{3}$ single crystal model, $\mathrm{I}^{-}$anions migrate along the $\mathrm{I}^{-}-\mathrm{I}^{-}$edge of the $\mathrm{PbI}_{6}$ octahedra when iodide vacancies appear; $\mathrm{MA}^{+}$cations migrate to its neighboring $\mathrm{MA}^{+}$vacancies along in the $<100>$ direction. ${ }^{136,144}$ These results indicate that decreasing the bulk defect densities in perovskite films has a significate effect on suppressing ion migration.

In addition to bulk defects, the surfaces and grain boundaries of the perovskite crystals are also important ion migration channels in perovskite films as shown in Fig. 4.2. Generally, perovskite films are polycrystalline films. Besides point defects, a large amount of surfaces and grain boundaries also exist in such films. Due to the relatively open structure, surfaces and grain boundaries exhibit small $E_{A}$ values. One experimental evidence for this is that a device based on a large-grain 


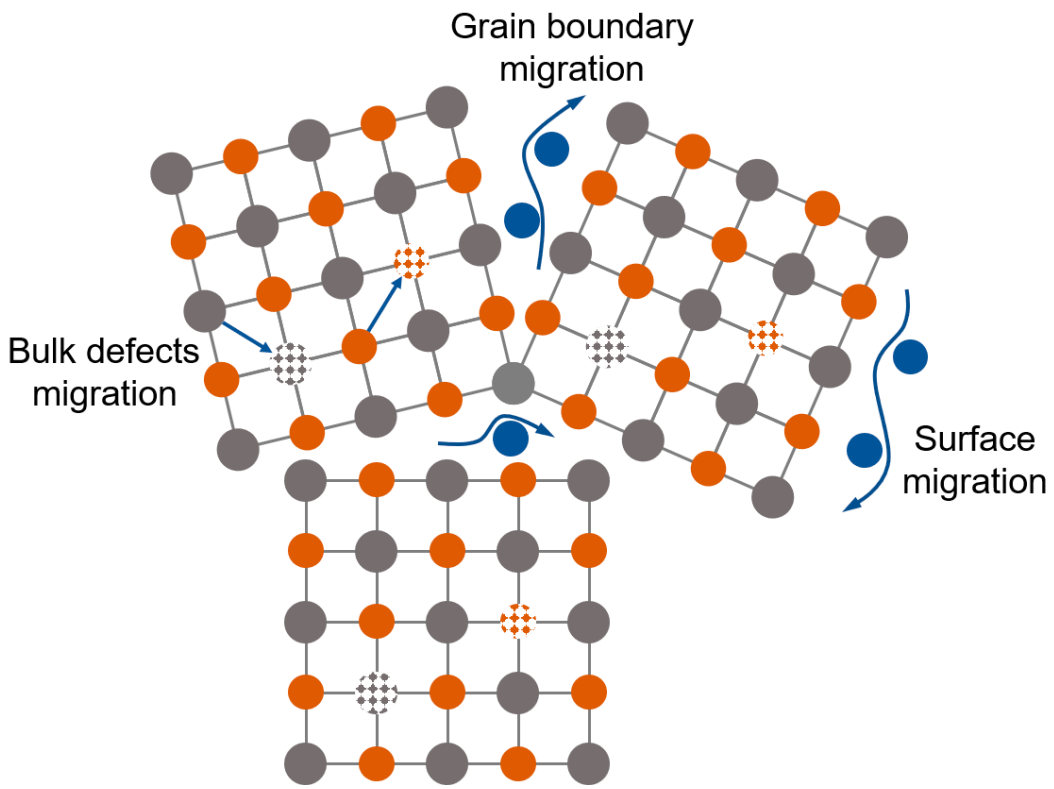

Figure 4.2 Ion migration channels in perovskite films.

perovskite film exhibits a difficult switchable photovoltaic effect, which is considered as the result of efficiently suppressed ion migration, compared with that of the device based on a small-grain perovskite film. ${ }^{145}$ Meanwhile, two kinds of ion movements were found in the perovskite polycrystalline films: "fast ions" moving under a small electrical filed (approximately $0.1 \mathrm{~V} \mathrm{\mu m}^{-1}$ ) and "slow ions" moving under a relatively high electrical field (over $0.3 \mathrm{~V} \mu \mathrm{m}^{-1}$ ). ${ }^{139}$ However, the origins of "fast ions" and "slow ions" movements have not been clearly identified. ${ }^{139}$ In addition, electrical field-induced $\operatorname{strain}^{146}$ and lightreduced binding between $\mathrm{MA}^{+}$and the inorganic frame ${ }^{147}$ in $\mathrm{MAPbI}_{3}$ have been demonstrated to promote ion migration in perovskite films.

\subsubsection{Evidence of Ion Migration}

To experimentally observe ion migration in metal halide perovskite materials, elemental (or functional groups) distribution of these materials have been investigated. Comparing the relative changes of elements (or functional groups) in the materials, the mobile-ion species and moving direction can be identified. Different technologies, such as time-of-flight 

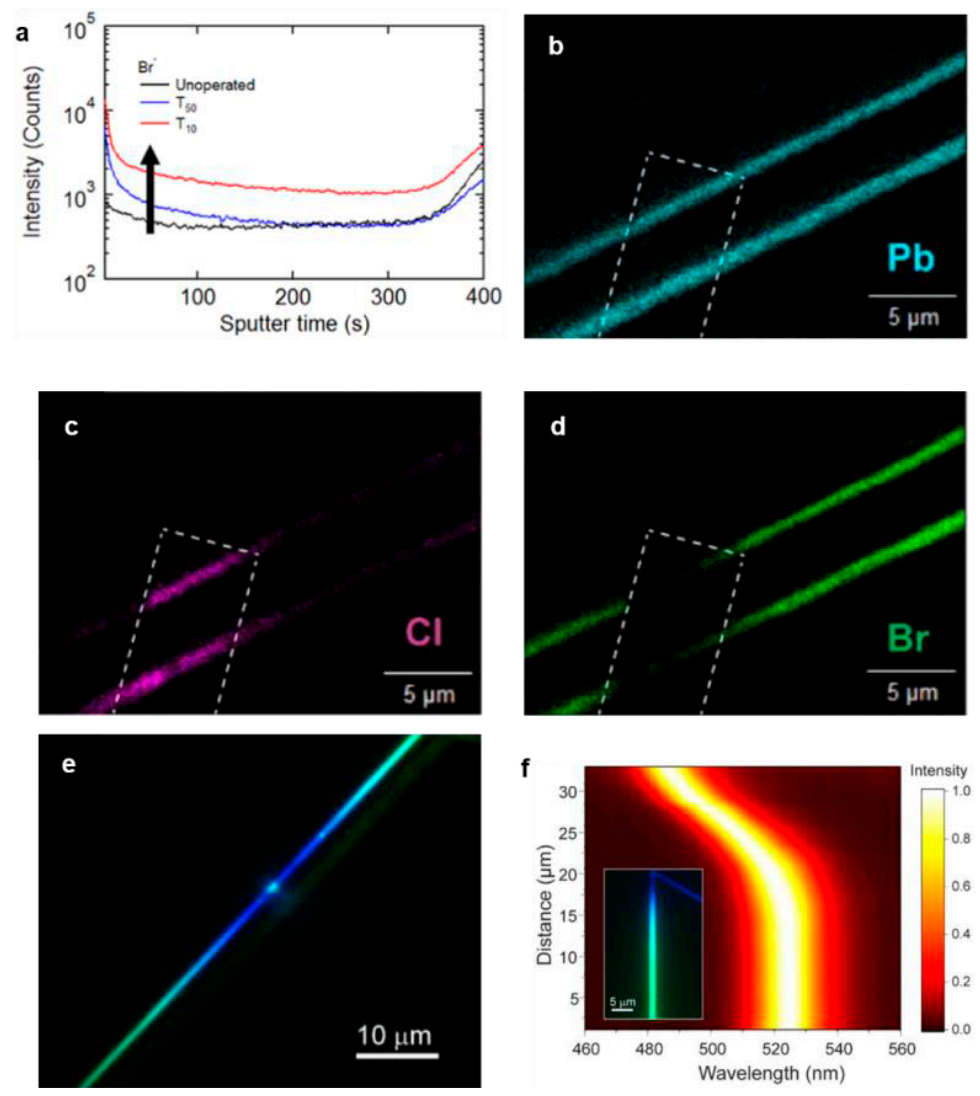

Figure 4.3 Evidence of ions migration. (a) Ion distribution of $\mathrm{Br}^{-}$anion with respect to the degradation time. ${ }^{129}$ (b-d) SEM-EDS mappings of the gradient nanowires show the spatial elemental distribution of $\mathrm{Pb}, \mathrm{Br}$, and $\mathrm{Cl}$, respectively. ${ }^{140}$ (e) Optical images of the $\mathrm{Cl}-\mathrm{Br}$ gradient nanowires. ${ }^{140}$ (f) Normalized PL line mapping of a gradient $\mathrm{CsPbBr}_{3-3 \mathrm{x}} \mathrm{Cl}_{3 \mathrm{x}}$ nanowire. The inset is a real-color PL image of the nanowire. ${ }^{140}$

secondary-ion mass spectrometry (TOF-SIMS) ${ }^{130,} 148$ and energydispersive X-ray (EDX) spectroscopy ${ }^{141}$, ${ }^{149}$, have been used to qualitatively identify the migration of mobile ions. For example, the TOFSIMS results showed clear evidence of the migration of $\mathrm{Br}^{-}$(as shown in Fig. 4.3a) and $\mathrm{CN}^{-}\left(\mathrm{MA}^{+}\right)$under electric field. ${ }^{130}$ A real-time luminance degradation image was observed in the perovskite film, which is ascribed to ion migration. ${ }^{130}$ Meanwhile, EDX spectroscopy showed the solidstate anion interdiffusion in $\mathrm{Cs}_{\mathrm{PbBr}} \mathrm{Br}_{3 \mathrm{x}} \mathrm{Cl}_{3 \mathrm{x}}$ nanowires (as shown in Fig. 
4.3b-d). ${ }^{141}$ In addition, the cross-sectional depth profile of iodine EDX spectroscopy suggested that electrode metal diffusion and iodine ion migration occur simultaneously during the aging process, and the iodine ions migrate toward the anode with a higher rate than metal diffusion through the hole transport layer. ${ }^{149}$

Fluorescence mapping is another technique to observe ion migration. The emission property of perovskites is directly related to the components of perovskites. Monitoring the emission variations under a constant measurement condition can reflect the movement of ions in the perovskite. As shown in Fig. 4.3b-f, ${ }^{141}$ the results of time-dependent confocal photoluminescence microscopy and energy-dispersive X-ray spectroscopy have quantitatively shown the solid-state anion interdiffusion in the $\mathrm{CsPb}\left(\mathrm{Br}_{1-\mathrm{x}} \mathrm{Cl}\right)_{3}$ perovskite nanowires, resulting in a halide concentration gradients. ${ }^{141}$ In addition, the X-ray fluorescence (XRF) mapping data provided the evidence of field-assisted ionic migration in the perovskite crystal and linked the optoelectronic properties with halide stoichiometry in the perovskite film. ${ }^{150}$ Ion migration and accumulation under external fields induce a quasireversible variation of halide-rich and halide-poor regions. ${ }^{150,151}$

\subsection{Ion Migration Induced Phenomena in PeLEDs}

Different experimental and theoretical results have confirmed the existence of ion migration in halide perovskite materials under an electrical field. Such ionic movements have been responsible for the commonly observed current-voltage hysteresis, ${ }^{152}$ switchable photovoltaic effects, ${ }^{145}$ and unsatisfactory stability ${ }^{135,143}$ in perovskite solar cells. Similar phenomena were also reported in PeLEDs, such as current-voltage hysteresis, ${ }^{7,64}$ electric field scan model related radiance and EQE, ${ }^{7,129}$ and poor stability. ${ }^{153-155}$ In this section, we discuss the impacts of ion migration on the efficiency and stability of PeLEDs.

\subsubsection{Efficiency}

As discussed in Chapter 1, the calculated EQEs are related to the number of injected carriers (current density) and the number of emitted photons (radiance). Therefore, in this section, we discuss EQE based on these two parameters. 

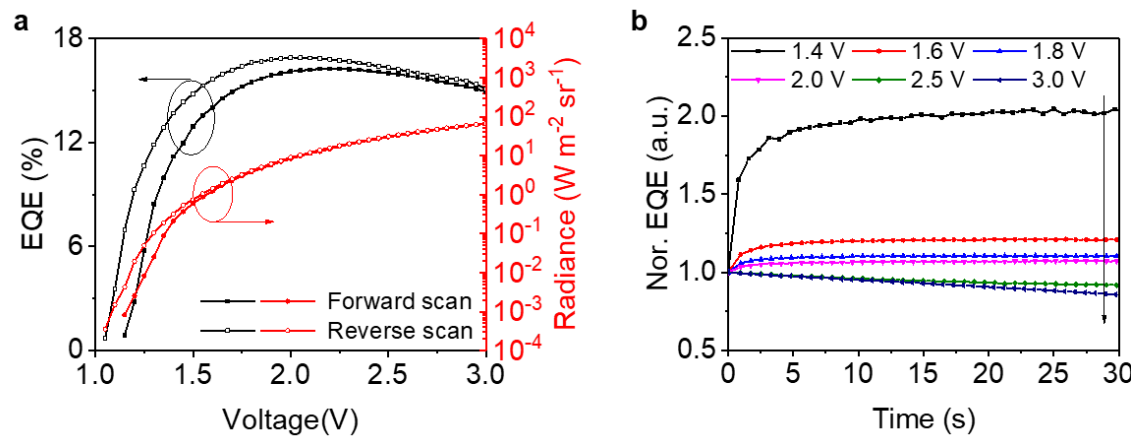

Figure 4.4 Ion migration induced enhancement of the EQE. (a) the EQEradiance-voltage hysteresis. (b) the initial enhanced EQE under constant applied driving voltages.

First, we discuss the current-voltage hysteresis in PeLEDs. As reported in the $\mathrm{MAPbI}_{3}$-based PeLEDs, ${ }^{7}$ compared with the forward scan (from low to high applied driving voltages), the devices show increased or decreased current densities at the same applied driving voltages during the reverse scan (from high to low applied driving voltages). The decreased current density at a relatively low applied driving voltage is induced by partially screened applied driving voltage as ion migration induces a new electric filed in the perovskite film. ${ }^{7}$ The increased current density at a relatively high applied driving voltage may be ascribed to the band bending at the interfacial layers between the perovskite layer and transport layers (Paper 5). In addition, the time-dependent current density and temperature-dependent current hysteresis were regarded as the result of ion migration. ${ }^{108,156}$

In addition to the change of carrier injection and transport in PeLEDs, ion migration also changes the emission property of perovskites. For example, as shown in Fig. 4.4a (Paper 5), the device shows both increased radiance and EQE during the reverse scan, which is similar to previous reports and is considered as the result of ion migration. ${ }^{64} \mathrm{In}$ addition, PeLEDs have shown both enhanced radiance and EQE during subsequent forward scans. ${ }^{129}$ As the same time, both steady-state photoluminescence (PL) intensity and time-resolved PL decay lifetime are increased after subsequent voltage scans, indicating the reduced nonradiative recombination in the perovskite film. ${ }^{129}$ The speculations of trap filling process $^{64}$ and traps driving out of emissive layer ${ }^{7}$ have been used to 
explain the enhanced EQE during the reverse scan; however, these trapping-related processes should occur within a short time (in the level of $\mu \mathrm{s}$ or $\mathrm{ms}$ ), in stark contrast to the long-term (several seconds or even longer) processes shown in Fig. 4.4b. Long-term enhanced EQE under constant applied driving voltages should be more likely ascribed to ion migration. Similar initial enhanced EQE under a constant current density was also observed, ${ }^{157}$ which is ascribed to the local migration of excess mobile ions.

\subsubsection{Stability}

The long-term stability has been recognized as one of the main problems hindering the commercialization of PeLEDs. In addition to extrinsic factors -such as thermal stress, air exposure, and illumination- ion migration in perovskite films is considered as an intrinsic reason for the unsatisfactory stability of PeLEDs. ${ }^{64}$ Ion migration in perovskites not only induces the fast degradation of PeLEDs, but also causes spectral instability in mixed halide perovskite systems.

Although ion migration sometimes could increase the initial EQE within a short time, it is still considered as the main reason for the fast degradation of PeLEDs. ${ }^{64}$ Under electrical stress, mobile ions can accumulate at the interfaces between the perovskite layer and other layers, contributing to the EL quenching and the poor operational stability of PeLEDs. ${ }^{158,159}$ In the meanwhile, the accumulated ions may cause the corrosion of metal electrodes. ${ }^{160}$ Besides the direct observation of ion migration as discussed above, some recent reports, which effectively suppress ion migration in the device, have resulted in improved operational stability. For example, reducing the ion migration channels by defects passivation leads to improved EQEs and operational stability of PeLEDs. ${ }^{62,84}$

In addition to the operational stability, ion migration can also affect the spectral stability. Mixing anions in the perovskite films is a significant strategy to tune the emission colors of PeLEDs. However, due to the different migration rates among various anions, mixed-halide perovskites even with uniform halide distribution can segregate into I-rich and $\mathrm{Br}-$ rich domains (or Br-rich and Cl-rich domains) under the illumination or electrical field ${ }^{153,154}$, known as phase segregation. The carriers undergo radiative recombination in I-rich and/or Br-rich domains (or Br-rich and/or Cl-rich domains), resulting in shifted emission peak or two 
emission peaks. ${ }^{143,}$ 161-163 Localized strain and defects have been demonstrated to promote ion migration and phase segregation. ${ }^{164,} 165$

\subsection{Suppress Ion Migration}

To achieve long-term operational stability of PeLEDs, minimizing ion migration is important to eliminate its negative effect. As discussed above, ion migration was affected by two factors, suitable migration channels and low $E_{A}$ value. Suppressing ion migration can thus be realized through regulating the migration channels and $E_{A}$ values of the perovskites.

Bulk defects, grain boundaries, and surfaces have been regarded as the main ion migration channels. Suppressing ion migration can be realized by decreasing the amount of these channels. High-quality perovskite films with high crystallinity, decreased bulk defects, and decreased grain boundaries have been demonstrated to effectively suppress ion migration, resulting in PeLEDs with improved EQE and operational stability. ${ }^{48,53}$ In addition, surface defect passivation by rational passivation agents is also beneficial to suppressing ion migration, resulting in improved operational stability of PeLEDs. For example, potassium bromide, ${ }^{166}$ amine, ${ }^{62,84}$ and large-sized cation ${ }^{64,167}$ have been demonstrated to assemble at the surface of perovskite materials, stopping the migration of mobile ions that come from inner parts of the materials. In addition, blocking materials can be used to suppress ion migration in PeLEDs, avoiding the degradation of charge transport layers or electrode caused by ion migration. The blocking materials can be mixed with perovskite nanocrystals ${ }^{168}$ or inserted between the perovskite layer and charge transport layers to improve the stability of PeLEDs. ${ }^{169}$

Increasing the $E_{A}$ values of the mobile ions is also useful to suppress ion migration. For example, potassium doped perovskites can increase the $E_{A}$ value of $\mathrm{I}^{-}$anions, increasing ion migration barriers in perovskite materials. ${ }^{170}$ In addition, partly replacing iodide anions by small-sized bromide anions also shows increased ion migration barriers. ${ }^{171}$ 



\section{Chapter 5 Outlook}

Although metal halide perovskite light-emitting diodes (PeLEDs) have attracted significant attention and shown rapid advances in the past several years, there are still challenges to be tackled before this promising technology can be implemented in commercial display and lighting applications. In this chapter, we focus on the critical challenges lying in the field of PeLEDs, such as high-performance blue, white, and lead-free PeLEDs. Additionally, we discuss possible strategies to address these challenges.

\subsection{High-Performance Blue PeLEDs}

PeLEDs with three primary colors, blue, green and red, are essential in display applications. However, the achievement of efficient blue PeLEDs remains a big challenge compared with their red and green counterparts. For instance, blue PeLEDs show lower external quantum efficiency (EQE) of $12.3 \%{ }^{172}$ than the green, red, and infrared PeLEDs, of which EQEs are over $20 \%{ }^{4-6}$

To realize blue emission, a straightforward approach is to modulate the composition of 3D perovskites. Partly replacing bromide $\left(\mathrm{Br}^{-}\right)$anion of bromide perovskites with small sized chloride $\left(\mathrm{Cl}^{-}\right)$anions can result in blue-shifted emission. ${ }^{173}$ However, as discussed in Chapter 4.2.2, mixed-halide perovskite films suffer from phase segregation, resulting in color instability during device operation. ${ }^{154}$ Although color instability is one big challenge for this strategy, there are promising methods to solve this issue. For example, proper defect passivation may help to stabilize the emission color by healing the ionic defects which mediate phase segregation. ${ }^{135}$ In addition, introducing small-sized cation (e.g. rubidium, $\left.\mathrm{Rb}^{+}\right)$in the $\mathrm{Cs} \mathrm{PbBr}_{3}$ perovskite films has resulted in blue emission, due to increased bandgap caused by the tilting of the $\left(\mathrm{PbBr}_{6}\right)^{4-}$ octahedra and the reduced overall orbital overlap. ${ }^{174,175}$

An alternative approach to realize blue emission is to introduce quantum confinement effects upon green perovskite emitters. However, a significant challenge for this strategy is the difficulty of size control. Generally, quantum confinement is obtained when the crystal size of a material is smaller than the Bohr diameter of this materials. The Bohr 
diameter of $\mathrm{CsPbr}_{3}$ is around $7 \mathrm{~nm} .{ }^{176}$ Abundant surface ligands are thus needed to stabilize such small perovskite QDs, however, the insulating nature of these ligands could result in poor charge injection and low brightness in PeLEDs. Alternatively, mixed-dimensional perovskites, which achieve quantum confinement in one or two dimensions, are another strategy to realize blue emission. ${ }^{177}$ However, as discussed in Chapter 3.2.2, efficient energy transfer from the large-bandgap phases to the low-bandgap phases occur in mixed-dimensional perovskite films, resulting in emissions from the perovskite phase of the lowest bandgap. ${ }^{178 \text {, }}$ 179 Therefore, achieving narrow distribution of $n$ values in the mixeddimensional perovskites is of critical importance to realize deep blue emission.

\subsection{White PeLEDs}

In addition to monochromatic emissions, perovskites have also demonstrated great opportunities for white light emission. White light is a combination of lights of different wavelengths, which can be generally described by Commission Internationale de L'Eclairage (CIE) coordinates. According to the Rec. 2020, the reference white light (D65) has CIE (1931) coordinates of (0.3127 0.3290). ${ }^{180}$

To realize white LEDs, one strategy is to mix three individual monochromatic emission lights by a proper ratio. At present, efficient hybrid LEDs combining perovskite color-down-conversion emitters with blue/ultraviolet (UV) chips have been developed. ${ }^{181,182}$ The chips emit blue/UV electroluminescence (EL) light, which excites green and red perovskite emitters to generate green and red emissions to form white light with the residual blue light.

White PeLEDs can also be fabricated by employing single emissive layers of orange polymer materials mixed with blue perovskite emitters. ${ }^{183}$ However, this kind of devices requires well controlled composition ratio between the blue and orange emissive species to fulfill balanced white light. Moreover, they usually show changed colors under different driving voltages/current densities.

PeLEDs with all-perovskite emitters have been developed through a multilayer device architecture. The white color is realized by mixing a red light and a cyan light, emitted by a two-dimensional perovskite and perovskite quantum dots, respectively. ${ }^{184}$ However, ion exchange between two perovskite layers is a big issue. The use of suitable 
interlayers is significant to avoid the ion exchange between two perovskite layers, as well as to balance the carrier transport and distribution in the device.

For lighting applications, a single emissive layer with efficient and stable white-light emission is preferred. Low-dimensional perovskites and doped double perovskites ${ }^{185-188}$ have been reported to show white light PL through the formation of self-trapped excitons, but the development of white PeLEDs based on these materials is barely reported. Both mechanism understanding and device engineering are desired to realize high-performance white PeLEDs.

\subsection{Lead-Free PeLEDs}

The toxicity of lead has raised significant concerns in metal halide perovskite-based devices. Although the lead content in perovskite-based optoelectronic devices is low enough to be safely used, the leakage of halide perovskites from devices into the environment still has a huge risk even with most rigorous encapsulation and the strictest recycling procedures. ${ }^{189}$ In addition, the lead from halide perovskites has been demonstrated to be more dangerous to the environment than other lead contamination sources already present in the ground. ${ }^{190}$ Therefore, increasing efforts should be made to develop other promising perovskite materials that are environmentally friendly and equally performing.

The development of lead-free PeLEDs is still at an early stage with only a few reports. 3D tin $\left(\mathrm{Sn}^{2+}\right)$-based perovskites $\left(\mathrm{CsSnI}_{3}\right)$ have been employed as emissive layers in lead-free PeLEDs. ${ }^{191}$ However, these perovskites suffer from poor stability in air. The zero-dimensional (0D) $\mathrm{Cs}_{3} \mathrm{Cu}_{2} \mathrm{I}_{5}$ exhibits good air-stability and high PLQY of $90 \%$, but the device performance of the resulting PeLEDs is poor. ${ }^{192}$ More efforts are desired on device engineering to improve the device performance.

Halide double perovskites and their derivatives are also promising materials for lead-free PeLEDs. ${ }^{193}$ For example, lead-free PeLEDs based on sodium (Na)-alloyed $\mathrm{Cs}_{2} \mathrm{AgInCl}_{6}$ double perovskites exhibit warmwhite emission. ${ }^{188}$ A range of different halide double perovskites have demonstrated high PLQYs; ${ }^{188,}$ 192, 194 however, the efficient LEDs based on such materials are rarely reported. A combination of experimental and theoretical investigations is desired to search for the right lead-free materials for high-performance LEDs. ${ }^{195}$ 



\section{References}

1 The electromagnetic spectrum, with the visible portion highlighted. https://en.wikipedia.org/wiki/Light\#/media/File:EM_spectrum.svg. Wikipedia (29 July 2020).

2 Young, T. The bakerian lecture. experiments and calculations relative to physical optics. Philos Trans $R$ Soc, 1832, 131-132 (1804).

3 Pulli, T., et al. Advantages of white LED lamps and new detector technology in photometry. Light Sci Appl, 4, 1-7 (2015).

4 Lin, K., et al. Perovskite light-emitting diodes with external quantum efficiency exceeding 20 per cent. Nature, 562, 245-248 (2018).

5 Cao, Y., et al. Perovskite light-emitting diodes based on spontaneously formed submicrometre-scale structures. Nature, 562, 249-253 (2018).

6 Chiba, T., et al. Anion-exchange red perovskite quantum dots with ammonium iodine salts for highly efficient light-emitting devices. Nat Photonics, 12, 681-687 (2018).

7 Tan, Z.-K., et al. Bright light-emitting diodes based on organometal halide perovskite. Nat Nanotechnol, 9, 687-692 (2014).

$8 \mathrm{Xu}, \mathrm{W}$., et al. Rational molecular passivation for high-performance perovskite light-emitting diodes. Nat Photonics, 13, 418-424 (2019).

9 Kathirgamanathan, P., et al. Electroluminescent organic and quantum dot LEDs: the state of the art. Journal of Display Technology, 11, 480-493 (2015).

10 Round, H. J. A note on carborundum. Electrical World, 49, 879879 (1907).

11 V., L. O. Luminous carborundum detector and detection effect and oscillations with crystals. The London, Edinburgh, and Dublin Philosophical Magazine and Journal of Science, 6, 1024-1044 (1928).

12 R, H. J., B, B. H. Radiation produced in germanium and silicon by electron-hole recombination. Bull Am Phys Soc, 86, 647-647 (1952).

13 J, K. R., M, Q. T. Recombination radiation emitted by gallium arsenide. Proc IRE, 50, 1822 (1962). 
14 Tang, C. W., VanSlyke, S. A. Organic electroluminescent diodes. Appl Phys Lett, 51, 913-915 (1987).

15 Yersin, H., et al. Design strategies for materials showing thermally activated delayed fluorescence and beyond: towards the fourthgeneration OLED mechanism. Journal of the SID, 26, 194-199 (2018).

16 Ai, X., et al. Efficient radical-based light-emitting diodes with doublet emission. Nature, 563, 536-540 (2018).

17 Tsujimura, T., et al. 45.1: Invited paper: Commercialization of world's first all - phosphorescent OLED product for lighting application. Dig Tech Pap, 43, 605-609 (2012).

18 Shirasaki, Y., et al. Emergence of colloidal quantum-dot lightemitting technologies. Nat Photonics, 7, 13-23 (2013).

19 Kim, J. Y., et al. 25th anniversary article: Colloidal quantum dot materials and devices: a quarter-century of advances. Adv Mater, 25, 4986-5010 (2013).

20 Spanhel, L., et al. Photochemistry of colloidal semiconductors. 20. Surface modification and stability of strong luminescing $\mathrm{CdS}$ particles. J Am Chem Soc, 109, 5649-5655 (1987).

21 Henglein, A. Small-particle research: physicochemical properties of extremely small colloidal metal and semiconductor particles. Chem Rev, 89, 1861-1873 (1989).

22 Steigerwald, M. L., Brus, L. E. Semiconductor crystallites: a class of large molecules. Acc Chem Res, 23, 183-188 (1990).

23 Murray, C. B., et al. Synthesis and characterization of nearly monodisperse $\mathrm{CdE}$ ( $\mathrm{E}=$ sulfur, selenium, tellurium) semiconductor nanocrystallites. J Am Chem Soc, 115, 8706-8715 (1993).

24 Peng, X., et al. Kinetics of II-VI and III-V colloidal semiconductor nanocrystal growth: "focusing" of size distributions. J Am Chem Soc, 120, 5343-5344 (1998).

25 Hines, M. A., Guyot-Sionnest, P. Synthesis and characterization of strongly luminescing ZnS-capped CdSe nanocrystals. J Phys Chem, 100, 468-471 (1996).

26 Reiss, P., et al. Highly luminescent $\mathrm{CdSe} / \mathrm{ZnSe}$ core/shell nanocrystals of low size dispersion. Nano Lett, 2, 781-784 (2002).

27 Mekis, I., et al. One-pot synthesis of highly luminescent $\mathrm{CdSe} / \mathrm{CdS}$ core- shell nanocrystals via organometallic and "Greener" chemical approaches. $J$ Phys Chem B, 107, 7454-7462 (2003). 
28 Ivanov, S. A., et al. Light amplification using inverted core/shell nanocrystals: towards lasing in the single-exciton regime. $J$ Phys Chem B, 108, 10625-10630 (2004).

29 Li, L., Reiss, P. One-pot synthesis of highly luminescent InP/ZnS nanocrystals without precursor injection. $J$ Am Chem Soc, 130, 11588-11589 (2008).

30 Peng, X., et al. Shape control of CdSe nanocrystals. Nature, 404, 59-61 (2000).

31 Manna, L., et al. Controlled growth of tetrapod-branched inorganic nanocrystals. Nat Mater, 2, 328-385 (2003).

32 Milliron, D. J., et al. Colloidal nanocrystal heterostructures with linear and branched topology. Nature, 430, 190-195 (2004).

33 Micic, O. I., et al. Synthesis and characterization of InP quantum dots. J Phys Chem, 98, 4966-4969 (1994).

34 Guzelian, A. A., et al. Synthesis of size-selected, surface-passivated InP nanocrystals. $J$ Phys Chem, 100, 7212-7219 (1996).

35 Murray, C. B., et al. Synthesis and characterization of monodisperse nanocrystals and close-packed nanocrystal assemblies. Annu Rev Mater Res, 30, 545-610 (2000).

36 Liang, J., et al. Recent progress and development in inorganic halide perovskite quantum dots for photoelectrochemical applications. Small, 16, 1-20 (2019).

37 Wells, H. L. Uber die casium-und kalium-bleihalogenide. Z Anorg Allg Chem, 3, 195-210 (1893).

38 Kojima, A., et al. Organometal halide perovskites as visible-light sensitizers for photovoltaic cells. J Am Chem Soc, 131, 6050-6051 (2009).

39 Amat, A., et al. Cation-induced band-gap tuning in organohalide perovskites: interplay of spin-orbit coupling and octahedra tilting. Nano Lett, 14, 3608-3616 (2014).

40 Eperon, G. E., et al. Formamidinium lead trihalide: a broadly tunable perovskite for efficient planar heterojunction solar cells. Energy Environ Sci, 7, 982-988 (2014).

41 Zhao, B., et al. High-efficiency perovskite-polymer bulk heterostructure light-emitting diodes. Nat Photonics, 12, 783-789 (2018).

42 Shen, Y., et al. High-efficiency perovskite light-emitting diodes with synergetic outcoupling enhancement. Adv Mater, 13, 1-8 (2019). 
43 Xing, G., et al. Transcending the slow bimolecular recombination in lead-halide perovskites for electroluminescence. Nat Commun, $\mathbf{8}$, 1-9 (2017).

44 Jin, H., et al. It's a trap! On the nature of localised states and charge trapping in lead halide perovskites. Mater Horiz, 7, 397-410 (2019).

45 Jeon, N. J., et al. Solvent engineering for high-performance inorganic-organic hybrid perovskite solar cells. Nat Mater, 13, 897903 (2014).

46 Miao, Y., et al. Stable and bright formamidinium-based perovskite light-emitting diodes with high energy conversion efficiency. Nat Commun, 10, 1-7 (2019).

$47 \mathrm{Wu}, \mathrm{C}$., et al. Improved performance and stability of all-inorganic perovskite light-emitting diodes by antisolvent vapor treatment. Adv Funct Mater, 27, 1-7 (2017).

$48 \mathrm{Yu}$, J. C., et al. Improving the stability and performance of perovskite light-emitting diodes by thermal annealing treatment. Adv Mater, 28, 6906-6913 (2016).

49 Pool, V. L., et al. Thermal engineering of $\mathrm{FAPbI}_{3}$ perovskite material via radiative thermal annealing and in situ XRD. Nat Commun, 8, 1-8 (2017).

50 Alsalloum, A. Y., et al. Low-temperature crystallization enables $21.9 \%$ efficient single-crystal $\mathrm{MAPbI}_{3}$ inverted perovskite solar cells. ACS Energy Letters, 5, 657-662 (2020).

51 Yang, W. S., et al. High-performance photovoltaic perovskite layers fabricated through intramolecular exchange. Science, 348, 1234-1237 (2015).

$52 \mathrm{Xu}, \mathrm{Z}$., et al. A thermodynamically favored crystal orientation in mixed formamidinium/methylammonium perovskite for efficient solar cells. Adv Mater, 31, 1-9 (2019).

53 Jia, Y. H., et al. Role of excess FAI in formation of high-efficiency $\mathrm{FAPbI}_{3}$-based light-emitting diodes. Adv Funct Mater, 30, 1-9 (2019).

54 Yan, K., et al. Hybrid halide perovskite solar cell precursors: colloidal chemistry and coordination engineering behind device processing for high efficiency. $J$ Am Chem Soc, 137, 4460-4468 (2015).

55 Qin, M., et al. Manipulating the mixed-perovskite crystallization pathway unveiled by in situ GIWAXS. Adv Mater, 1, 1-10 (2019). 
56 Wang, H., et al. Perovskite-molecule composite thin films for efficient and stable light-emitting diodes. Nat Commun, 11, 1-9 (2020).

57 Feng, J., et al. Record efficiency stable flexible perovskite solar cell using effective additive assistant strategy. Adv Mater, 30, 1-9 (2018).

58 Shamsi, J., et al. Metal halide perovskite nanocrystals: synthesis, post-synthesis modifications, and their optical properties. Chem Rev, 119, 3296-3348 (2019).

59 Yang, X., et al. Efficient green light-emitting diodes based on quasi-two-dimensional composition and phase engineered perovskite with surface passivation. Nat Commun, 9, 1-8 (2018).

60 Lee, S., et al. Versatile defect passivation methods for metal halide perovskite materials and their application to light-emitting devices. Adv Mater, 31, 1-17 (2019).

61 Chen, J.-K., et al. High-efficiency violet-emitting all-inorganic perovskite nanocrystals enabled by alkaline-earth metal passivation. Chem Mater, 31, 3974-3983 (2019).

62 Lee, S., et al. Growth of nanosized single crystals for efficient perovskite light-emitting diodes. ACS Nano, 12, 3417-3423 (2018).

63 Cho, H., et al. Overcoming the electroluminescence efficiency limitations of perovskite light-emitting diodes. Science, 350, 12221225 (2015).

64 Xiao, Z., et al. Efficient perovskite light-emitting diodes featuring nanometre-sized crystallites. Nat Photonics, 11, 108-115 (2017).

$65 \mathrm{Li}, \mathrm{G}$., et al. Efficient light-emitting diodes based on nanocrystalline perovskite in a dielectric polymer matrix. Nano Lett, 15, 2640-2644 (2015).

66 Feldmann, S., et al. Photodoping through local charge carrier accumulation in alloyed hybrid perovskites for highly efficient luminescence. Nat Photonics, 14, 123-128 (2019).

67 Nenon, D. P., et al. Design principles for trap-free $\mathrm{CsPbX}_{3}$ nanocrystals: enumerating and eliminating surface halide vacancies with softer lewis bases. J Am Chem Soc, 140, 17760-17772 (2018).

$68 \mathrm{Fu}, \mathrm{Y}$., et al. Metal halide perovskite nanostructures for optoelectronic applications and the study of physical properties. Nat Rev Mater, 4, 169-188 (2019).

69 Gao, P., et al. Dimensionality engineering of hybrid halide perovskite light absorbers. Nat Commun, 9, 1-14 (2018). 
70 Chen, Y., et al. 2D Ruddlesden-Popper perovskites for optoelectronics. Adv Mater, 30, 1-15 (2018).

71 Verzellesi, G., et al. Efficiency droop in InGaN/GaN blue lightemitting diodes: Physical mechanisms and remedies. $J$ Appl Phys, 114, 1-14 (2013).

72 Bae, W. K., et al. Controlling the influence of Auger recombination on the performance of quantum-dot light-emitting diodes. Nat Commun, 4, 1-8 (2013).

73 Zou, W., et al. Minimising efficiency roll-off in high-brightness perovskite light-emitting diodes. Nat Commun, 9, 1-7 (2018).

74 Fakharuddin, A., et al. Reduced efficiency roll-off and improved stability of mixed 2D/3D perovskite light emitting diodes by balancing charge injection. Adv Funct Mater, 29, 1-12 (2019).

75 Meng, Y., et al. High performance and stable all-inorganic perovskite light emitting diodes by reducing luminescence quenching at PEDOT:PSS/perovskites interface. Org Electron, 64, 47-53 (2019).

76 Zhang, X., et al. Enhancing the brightness of cesium lead halide perovskite nanocrystal based green light-emitting devices through the interface engineering with perfluorinated ionomer. Nano Lett, 16, 1415-1420 (2016).

77 Kim, Y. H., et al. Multicolored organic/inorganic hybrid perovskite light-emitting diodes. Adv Mater, 27, 1248-1254 (2015).

78 Zou, Y., et al. Boosting perovskite light-emitting diode performance via tailoring interfacial contact. ACS Appl Mater Interfaces, 10, 24320-24326 (2018).

79 Ahn, Y., et al. Improving the efficiency of perovskite light emitting diode using polyvinylpyrrolidone as an interlayer. Appl Surf Sci, 507, 1-7 (2020).

80 Chih, Y. K., et al. $\mathrm{NiO}_{\mathrm{x}}$ electrode interlayer and $\mathrm{CH}_{3} \mathrm{NH}_{2} / \mathrm{CH}_{3} \mathrm{NH}_{3} \mathrm{PbBr}_{3}$ interface treatment to markedly advance hybrid perovskite-based light-emitting diodes. Adv Mater, 28, 8687-8694 (2016).

81 Wang, J., et al. Interfacial control toward efficient and low-voltage perovskite light-emitting diodes. Adv Mater, 27, 2311-2316 (2015).

82 Zhang, L., et al. Ultra-bright and highly efficient inorganic based perovskite light-emitting diodes. Nat Commun, 8, 1-8 (2017). 
83 Yuan, Z., et al. Unveiling the synergistic effect of precursor stoichiometry and interfacial reactions for perovskite light-emitting diodes. Nat Commun, 10, 1-9 (2019).

84 Lee, S., et al. Amine-based passivating materials for enhanced optical properties and performance of organic-inorganic perovskites in light-emitting diodes. $J$ Phys Chem Lett, 8, 17841792 (2017).

85 Shi, X.-B., et al. Optical energy losses in organic-inorganic hybrid perovskite light-emitting diodes. Adv Opt Mater, 6, 1-7 (2018).

86 Zhao, L., et al. Improved outcoupling efficiency and stability of perovskite light-emitting diodes using thin emitting layers. $A d v$ Mater, 31, 1-6 (2019).

87 Zhang, Q., et al. Efficient metal halide perovskite light-emitting diodes with significantly improved light extraction on nanophotonic substrates. Nat Commun, 10, 1-9 (2019).

$88 \mathrm{Gao}, \mathrm{Y}$., et al. Ultrathin $\mathrm{CsPbX}_{3}$ nanowire arrays with strong emission anisotropy. Adv Mater, 30, 1-9 (2018).

89 Jurow, M. J., et al. Tunable anisotropic photon emission from selforganized $\mathrm{CsPbBr}_{3}$ perovskite nanocrystals. Nano Lett, 17, 45344540 (2017).

90 Quan, L. N., et al. Perovskites for next-generation optical sources. Chem Rev, 119, 7444-7477 (2019).

91 Huang, H., et al. Colloidal lead halide perovskite nanocrystals: synthesis, optical properties and applications. NPG Asia Materials, 8, 1-15 (2016).

92 Min, X., et al. Research progress of low-dimensional perovskites: synthesis, properties and optoelectronic applications. J Semicond, 38, 1-9 (2017).

93 Bai, S., et al. Planar perovskite solar cells with long-term stability using ionic liquid additives. Nature, 571, 245-250 (2019).

94 Dunlap-Shohl, W. A., et al. Synthetic approaches for halide perovskite thin films. Chem Rev, 119, 3193-3295 (2019).

95 Tiep, N. H., et al. Recent advances in improving the stability of perovskite solar cells. Adv Energy Mater, 6, 1-19 (2016).

96 Qing, J., et al. Aligned and graded type-II Ruddlesden-Popper perovskite films for efficient solar cells. Adv Energy Mater, 8, 1-8 (2018). 
97 Chen, P., et al. In situ growth of 2D perovskite capping layer for stable and efficient perovskite solar cells. Adv Funct Mater, 28, 110 (2018).

98 Venables, J. A., Spiller, G. D. T. Nucleation and growth of thin films. Surface Mobilities on Solid Materials, 86, 341-404 (1983).

99 Hiroshi, M., et al. The crystal structure of lead (II) iodidedimethylsulphoxide (1/2), $\mathrm{PbI}_{2}$ (dmso) 2 . Chem Lett, 9, 663-664 (1980).

100 Mao, W., et al. Controlled growth of monocrystalline organo-lead halide perovskite and its application in photonic devices. Angew Chem Int Ed Engl, 56, 12486-12491 (2017).

101 Song, Z., et al. Impact of processing temperature and composition on the formation of methylammonium lead iodide perovskites. Chem Mater, 27, 4612-4619 (2015).

102 Zheng, X., et al. Dual functions of crystallization control and defect passivation enabled by sulfonic zwitterions for stable and efficient perovskite solar cells. Adv Mater, 30, 1-8 (2018).

$103 \mathrm{Li}$, B., et al. Engineering halide perovskite crystals through precursor chemistry. Small, 15, 1-24 (2019).

104 Ava, T. T., et al. A review: thermal stability of methylammonium lead halide based perovskite solar cells. Appl Sci, 9, 1-25 (2019).

105 Aleksanyan, E., et al. Mechanisms of mechanochemical synthesis of cesium lead halides: pathways toward stabilization of $\alpha-\mathrm{CsPb}_{3}$. J Mater Sci, 55, 8665-8678 (2020).

106 Liu, T., et al. High-performance formamidinium-based perovskite solar cells via microstructure-mediated $\delta$-to- $\alpha$ phase transformation. Chem Mater, 29, 3246-3250 (2017).

107 Brennan, M. C., et al. Photoinduced anion segregation in mixed halide perovskites. Trends in Chemistry, 2, 282-301 (2020).

108 Cho, H., et al. High-efficiency solution-processed inorganic metal halide perovskite light-emitting diodes. Adv Mater, 29, 1-8 (2017).

109 Stamplecoskie, K. G., et al. Dual nature of the excited state in organic-inorganic lead halide perovskites. Energy Environ Sci, 8, 208-215 (2015).

110 Zhao, X., et al. Simple and efficient green-light-emitting diodes based on thin organolead bromide perovskite films via tuning precursor ratios and postannealing temperature. J Phys Chem Lett, 7, 4259-4266 (2016). 
111 Lee, J.-W., et al. In-situ formed type I nanocrystalline perovskite film for highly efficient light-emitting diode. ACS Nano, 11, 33113319 (2017).

112 Wei, Z., et al. Solution-processed highly bright and durable cesium lead halide perovskite light-emitting diodes. Nanoscale, 8, 1802118026 (2016).

113 Ma, F., et al. Stable alpha/delta phase junction of formamidinium lead iodide perovskites for enhanced near-infrared emission. Chem Sci, 8, 800-805 (2017).

114 Chen, Z., et al. High-performance color-tunable perovskite light emitting devices through structural modulation from bulk to layered film. Adv Mater, 29, 1-8 (2017).

115 Qing, J., et al. High-quality ruddlesden-popper perovskite films based on in situ formed organic spacer cations. Adv Mater, 31, 1-8 (2019).

116 Cheng, L.-P., et al. Efficient $\mathrm{Cs}_{\mathrm{PbBr}}$ perovskite light-emitting diodes enabled by synergetic morphology control. Adv Opt Mater, 7, 1-9 (2019).

117 Ban, M., et al. Solution-processed perovskite light emitting diodes with efficiency exceeding $15 \%$ through additive-controlled nanostructure tailoring. Nat Commun, 9, 1-10 (2018).

118 Fang, Z., et al. Dual passivation of perovskite defects for lightemitting diodes with external quantum efficiency exceeding $20 \%$. Adv Funct Mater, 30, 1-9 (2020).

119 Park, M. H., et al. Efficient perovskite light-emitting diodes using polycrystalline core-shell-mimicked nanograins. Adv Funct Mater, 29, 1-19 (2019).

$120 \mathrm{Yu}, \mathrm{F} . \mathrm{X}$., et al. Full coverage all-inorganic cesium lead halide perovskite film for high-efficiency light-emitting diodes assisted by 1,3,5-tri(m-pyrid-3-yl-phenyl)benzene. Org Electron, 50, 480-484 (2017).

121 Park, M.-H., et al. Unravelling additive-based nanocrystal pinning for high efficiency organic-inorganic halide perovskite lightemitting diodes. Nano Energy, 42, 157-165 (2017).

122 La-Placa, M. G., et al. Photoluminescence quantum yield exceeding $80 \%$ in low dimensional perovskite thin-films via passivation control. Chem Commun (Camb), 53, 8707-8710 (2017). 
123 Aristidou, N., et al. Fast oxygen diffusion and iodide defects mediate oxygen-induced degradation of perovskite solar cells. Nat Commun, 8, 1-10 (2017).

124 Tsai, H., et al. Light-induced lattice expansion leads to highefficiency perovskite solar cells. Sicience, 360, 67-70 (2018).

125 Ahn, N., et al. Trapped charge-driven degradation of perovskite solar cells. Nat Commun, 7, 1-9 (2016).

$126 \mathrm{Li}, \mathrm{M}$., et al. Pressure responses of halide perovskites with various compositions, dimensionalities, and morphologies. Matter Radiat Extremes, 5, 1-15 (2020).

127 Jamaludin, N. F., et al. Grain size modulation and interfacial engineering of $\mathrm{CH}_{3} \mathrm{NH}_{3} \mathrm{PbBr}_{3}$ emitter films through incorporation of tetraethylammonium bromide. Chemphyschem, 19, 1075-1080 (2018).

128 deQuilettes, D. W., et al. Photo-induced halide redistribution in organic-inorganic perovskite films. Nat Commun, 7, 1-9 (2016).

129 Zhao, L., et al. Electrical stress influences the efficiency of $\mathrm{CH}_{3} \mathrm{NH}_{3} \mathrm{PbI}_{3}$ perovskite light emitting devices. Adv Mater, 29, 1-6 (2017).

130 Lee, H., et al. Direct evidence of ion-migration-induced degradation of ultrabright perovskite light-emitting diodes. ACS Appl Mater Interfaces, 11, 11667-11673 (2019).

131 Inaguma, Y., et al. Lithium ion conductivity in the perovskite-type $\mathrm{LiTaO}_{3}-\mathrm{SrTiO}_{3}$ solid solution. Solid State Ion, 79, 91-97 (1995).

132 Ishihara, $\mathrm{T}$., et al. Doped $\mathrm{LaGaO}_{3}$ perovskite type oxide as a new oxide ionic conductor. J Am Chem Soc, 116, 3801-3803 (1994).

133 Yang, T. Y., et al. The significance of ion conduction in a hybrid organic-inorganic lead-iodide-based perovskite photosensitizer. Angew Chem Int Ed Engl, 127, 8016-8021 (2015).

134 Calado, P., et al. Evidence for ion migration in hybrid perovskite solar cells with minimal hysteresis. Nat Commun, 7, 1-10 (2016).

135 Yuan, Y., Huang, J. Ion migration in organometal trihalide perovskite and its impact on photovoltaic efficiency and stability. Acc Chem Res, 49, 286-293 (2016).

136 Eames, C., et al. Ionic transport in hybrid lead iodide perovskite solar cells. Nat Commun, 6, 1-8 (2015).

137 Haruyama, J., et al. First-principles study of ion diffusion in perovskite solar cell sensitizers. J Am Chem Soc, 137, 10048-10051 (2015). 
138 Yin, W.-J., et al. Unusual defect physics in $\mathrm{CH}_{3} \mathrm{NH}_{3} \mathrm{PbI}_{3}$ perovskite solar cell absorber. Appl Phys Lett, 104, 1-4 (2014).

139 Yuan, Y., et al. Photovoltaic switching mmechanism in lateral structure hybrid perovskite solar cells. Adv Energy Mater, 5, 1-7 (2015).

$140 \mathrm{Li}$, D., et al. Effective approach for reducing the migration of ions and improving the stability of organic-inorganic perovskite solar cells. J Alloys Compd, 741, 489-494 (2018).

141 Pan, D., et al. Visualization and studies of ion-diffusion kinetics in cesium lead bromide perovskite nanowires. Nano Lett, 18, 18071813 (2018).

142 Egger, D. A., et al. Theory of hydrogen migration in organicinorganic halide perovskites. Angew Chem Int Ed Engl, 54, 1243712441 (2015).

143 Lin, Y., et al. Excess charge-carrier induced instability of hybrid perovskites. Nat Commun, 9, 1-9 (2018).

$144 \mathrm{Kim}, \mathrm{J}$, et al. The role of intrinsic defects in methylammonium lead iodide perovskite. J Phys Chem Lett, 5, 1312-1317 (2014).

145 Xiao, Z., et al. Giant switchable photovoltaic effect in organometal trihalide perovskite devices. Nat Mater, 14, 193-198 (2015).

146 Dong, Q., et al. Lateral-structure single-crystal hybrid perovskite solar cells via piezoelectric poling. Adv Mater, 28, 2816-2821 (2016).

147 Gottesman, R., et al. Extremely slow photoconductivity response of $\mathrm{CH}_{3} \mathrm{NH}_{3} \mathrm{PbI}_{3}$ perovskites suggesting structural changes under working conditions. J Phys Chem Lett, 5, 2662-2669 (2014).

$148 \mathrm{Li}, \mathrm{Z}$., et al. Extrinsic ion migration in perovskite solar cells. Energy Environ Sci, 10, 1234-1242 (2017).

149 Liu, L., et al. Grain-boundary "patches" by in situ conversion to enhance perovskite solar cells stability. Adv Mater, 30, 1-8 (2018).

150 Luo, Y., et al. Direct observation of halide migration and its effect on the photoluminescence of methylammonium lead bromide perovskite single crystals. Adv Mater, 29, 1-7 (2017).

$151 \mathrm{Li}, \mathrm{C}$. , et al. Real-time observation of iodide ion migration in methylammonium lead halide perovskites. Small, 13, 1-10 (2017).

152 Snaith, H. J., et al. Anomalous hysteresis in perovskite solar cells. J Phys Chem Lett, 5, 1511-1515 (2014). 
153 Hoke, E. T., et al. Reversible photo-induced trap formation in mixed-halide hybrid perovskites for photovoltaics. Chem Sci, 6, 613-617 (2015).

$154 \mathrm{Li}, \mathrm{G}$., et al. Highly efficient perovskite nanocrystal light-emitting diodes enabled by a universal crosslinking method. Adv Mater, 28, 3528-3534 (2016).

155 Chen, M., et al. Manipulating ion migration for highly stable lightemitting diodes with single-crystalline organometal halide perovskite microplatelets. ACS Nano, 11, 6312-6318 (2017).

$156 \mathrm{Li}, \mathrm{C}$., et al. Unravelling the role of vacancies in lead halide perovskite through electrical switching of photoluminescence. Nat Commun, 9, 1-8 (2018).

157 Cho, H., et al. Improving the stability of metal halide perovskite materials and light-emitting diodes. Adv Mater, 30, 1-24 (2018).

158 Qin, J., et al. Enabling self-passivation by attaching small grains on surfaces of large grains toward high-performance perovskite LEDs. iScience, 19, 378-387 (2019).

159 Prakasam, V., et al. Degradation mechanisms in organic lead halide perovskite light-emitting diodes. Adv Opt Mater, 7, 1-7 (2019).

160 Guo, Y., et al. Degradation mechanism of perovskite light-emitting diodes: an in situ investigation via electroabsorption spectroscopy and device modelling. Adv Funct Mater, 30, 1-8 (2020).

161 Draguta, S., et al. Rationalizing the light-induced phase separation of mixed halide organic-inorganic perovskites. Nat Commun, 8 , 18 (2017).

162 Kumawat, N. K., et al. Blue perovskite light-emitting diodes: progress, challenges and future directions. Nanoscale, 11, 21092120 (2019).

163 Vashishtha, P., Halpert, J. E. Field-driven ion migration and color instability in red-emitting mixed halide perovskite nanocrystal light-emitting diodes. Chem Mater, 29, 5965-5973 (2017).

164 Bischak, C. G., et al. Origin of reversible photoinduced phase separation in hybrid perovskites. Nano Lett, 17, 1028-1033 (2017).

165 Barker, A. J., et al. Defect-assisted photoinduced halide segregation in mixed-halide perovskite thin films. ACS Energy Letters, 2, 14161424 (2017).

166 Yang, J. N., et al. Potassium-bromide surface passivation on $\mathrm{CsPbI}_{3-\mathrm{x}} \mathrm{Br}_{\mathrm{x}}$ nanocrystals for efficient and stable pure red perovskite light emitting diodes. J Am Chem Soc, 142, 2956-2967 (2020). 
167 Xiao, Z., et al. Mixed-halide perovskites with stabilized bandgaps. Nano Lett, 17, 6863-6869 (2017).

168 Wang, X., et al. Suppressed phase separation of mixed-halide perovskites confined in endotaxial matrices. Nat Commun, 10, 1-7 (2019).

$169 \mathrm{Li}, \mathrm{C}$., et al. Understanding the improvement in the stability of a self-assembled multiple-quantum well perovskite light-emitting diode. J Phys Chem Lett, 10, 6857-6864 (2019).

170 Cao, J., et al. Interstitial occupancy by extrinsic alkali cations in perovskites and its impact on ion migration. Adv Mater, 30, 1-9 (2018).

171 Zhang, T., et al. Understanding the relationship between ion migration and the anomalous hysteresis in high-efficiency perovskite solar cells: a fresh perspective from halide substitution. Nano Energy, 26, 620-630 (2016).

172 Dong, Y., et al. Bipolar-shell resurfacing for blue LEDs based on strongly confined perovskite quantum dots. Nat Nanotechnol, 15, 668-674 (2020).

173 Liu, R., Xu, K. Blue perovskite light-emitting diodes (LEDs): A minireview. Instrum Sci Technol, 48, 619-636 (2020).

174 Linaburg, M. R., et al. $\mathrm{Cs}_{1-\mathrm{x}} \mathrm{Rb}_{\mathrm{x}} \mathrm{PbCl}_{3}$ and $\mathrm{Cs}_{1_{-\mathrm{x}}} \mathrm{Rb}_{\mathrm{x}} \mathrm{PbBr}_{3}$ solid solutions: understanding octahedral tilting in lead halide perovskites. Chem Mater, 29, 3507-3514 (2017).

175 Jiang, Y., et al. Spectra stable blue perovskite light-emitting diodes. Nat Commun, 10, 1-9 (2019).

176 Protesescu, L., et al. Nanocrystals of cesium clead halide perovskites $(\mathrm{CsPbX}(3), \mathrm{X}=\mathrm{Cl}, \mathrm{Br}$, and I): novel optoelectronic materials showing bright emission with wide color gamut. Nano Lett, 15, 3692-3696 (2015).

177 Liu, Y., et al. Efficient blue light-emitting diodes based on quantum-confined bromide perovskite nanostructures. Nat Photonics, 13, 760-764 (2019).

178 Wang, N., et al. Perovskite light-emitting diodes based on solutionprocessed self-organized multiple quantum wells. Nat Photonics, 10, 699-704 (2016).

179 Yuan, M., et al. Perovskite energy funnels for efficient lightemitting diodes. Nat Nanotechnol, 11, 872-877 (2016). 
180 "BT.2020: Parameter values for ultra-high definition television systems for production and international programme exchange". International Telecommunication Union. Approved in 2015-10.

181 Di, X., et al. Efficient white LEDs with bright green-emitting $\mathrm{CsPbBr}_{3}$ perovskite nanocrystal in mesoporous silica nanoparticles. J Alloys Compd, 729, 526-532 (2017).

182 Pan, A., et al. Stable luminous nanocomposites of $\mathrm{CsPbX}_{3}$ perovskite nanocrystals anchored on silica for multicolor anticounterfeit ink and white-LEDs. Mater Chem Front, 3, 414-419 (2019).

183 Yao, E. P., et al. High-brightness blue and white LEDs based on inorganic perovskite nanocrystals and their composites. Adv Mater, 29, 1-7 (2017).

184 Mao, J., et al. All-perovskite emission architecture for white lightemitting diodes. ACS Nano, 12, 10486-10492 (2018).

185 Dohner, E. R., et al. Self-assembly of broadband white-light emitters. J Am Chem Soc, 136, 1718-1721 (2014).

186 Dohner, E. R., et al. Intrinsic white-light emission from layered hybrid perovskites. J Am Chem Soc, 136, 13154-13157 (2014).

187 Yangui, A., et al. Optical investigation of broadband white-light emission in self-assembled organic-inorganic perovskite $\left(\mathrm{C}_{6} \mathrm{H}_{11} \mathrm{NH}_{3}\right)_{2} \mathrm{PbBr}_{4}$. J Phys Chem C, 119, 23638-23647 (2015).

188 Luo, J., et al. Efficient and stable emission of warm-white light from lead-free halide double perovskites. Nature, 563, 541-545 (2018).

189 Jiang, Y., et al. Reduction of lead leakage from damaged lead halide perovskite solar modules using self-healing polymer-based encapsulation. Nature Energy, 4, 585-593 (2019).

$190 \mathrm{Li}$, J., et al. Biological impact of lead from halide perovskites reveals the risk of introducing a safe threshold. Nat Commun, 11, 1-5 (2020).

191 Hong, W. L., et al. Efficient low-temperature solution-processed lead-free perovskite infrared light-emitting diodes. Adv Mater, 28, 8029-8036 (2016).

192 Jun, T., et al. Lead-free highly efficient blue-emitting $\mathrm{Cs}_{3} \mathrm{Cu}_{2} \mathrm{I}_{5}$ with 0D electronic structure. Adv Mater, 30, 1-6 (2018).

193 Ning, W., Gao, F. Structural and functional diversity in lead-free halide perovskite materials. Adv Mater, 31, 1-21 (2019). 
References

194 Ma, Z., et al. Electrically-driven violet light-emitting devices based on highly stable lead-free perovskite $\mathrm{Cs}_{3} \mathrm{Sb}_{2} \mathrm{Br}_{9}$ quantum dots. $A C S$ Energy Letters, 5, 385-394 (2019).

$195 \mathrm{Lu}$, S., et al. Accelerated discovery of stable lead-free hybrid organic-inorganic perovskites via machine learning. Nat Commun, 9, 1-8 (2018). 



\section{Papers}

The papers associated with this thesis have been removed for copyright reasons. For more details about these see:

http://urn.kb.se/resolve?urn=urn:nbn:se:liu:diva-169447 


\section{FACULTY OF SCIENCES AND ENGINEERING}

Linköping Studies in Science and Technology. Dissertations, No. 2086, 2020

Department of Physics, Chemistry and Biology (IFM)

Linköping University

SE-581 83 Linköping, Sweden

www.liu.se 